ANALYSIS \& PDE $\begin{array}{lll}\text { Volume } 8 & \text { No. } 3 \quad 2015\end{array}$ CHe JOSE M. CONDE-ALONSO, TAO MEI AND JAVIER PARCET

\title{
LARGE BMO SPACES VS INTERPOLATION
}




\title{
LARGE BMO SPACES VS INTERPOLATION
}

\author{
Jose M. Conde-Alonso, Tao Mei And JaVier Parcet
}

\begin{abstract}
We introduce a class of BMO spaces which interpolate with $L_{p}$ and are sufficiently large to serve as endpoints for new singular integral operators. More precisely, let $(\Omega, \Sigma, \mu)$ be a $\sigma$-finite measure space. Consider two filtrations of $\Sigma$ by successive refinement of two atomic $\sigma$-algebras $\Sigma_{\mathrm{a}}$ and $\Sigma_{\mathrm{b}}$ having trivial intersection. Construct the corresponding truncated martingale BMO spaces. Then, the intersection seminorm only leaves out constants and we provide a quite flexible condition on $\left(\Sigma_{\mathrm{a}}, \Sigma_{\mathrm{b}}\right)$ so that the resulting space interpolates with $L_{p}$ in the expected way. In the presence of a metric $d$, we obtain endpoint estimates for Calderón-Zygmund operators on $(\Omega, \mu, d)$ under additional conditions on $\left(\Sigma_{\mathrm{a}}, \Sigma_{\mathrm{b}}\right)$. These are weak forms of the "isoperimetric" and the "locally doubling" properties of Carbonaro, Mauceri and Meda which admit less concentration at the boundary. Examples of particular interest include densities of the form $e^{ \pm|x|^{\alpha}}$ for any $\alpha>0$ or $\left(1+|x|^{\beta}\right)^{-1}$ for any $\beta \gtrsim n^{3 / 2}$. A (limited) comparison with Tolsa's RBMO is also possible. On the other hand, a more intrinsic formulation yields a Calderón-Zygmund theory adapted to regular filtrations over $\left(\Sigma_{\mathrm{a}}, \Sigma_{\mathrm{b}}\right)$ without using a metric. This generalizes well-known estimates for perfect dyadic and Haar shift operators. In contrast to previous approaches, ours extends to matrix-valued functions (via recent results from noncommutative martingale theory) for which only limited results are known and no satisfactory nondoubling theory exists so far.
\end{abstract}

\section{Introduction}

A BMO space is a set of functions that enjoy bounded mean oscillation in a certain sense. Both "mean" and "oscillation" can be measured in many different ways. Most frequently, we find BMO spaces refer to averages over balls in a metric measure space. In other notable scenarios, we may replace these averages by conditional expectations with respect to a martingale filtration, or even by the action of a nicely behaved semigroup of operators. These more abstract formulations are known to be very useful given the lack of appropriate metrics. The relation between metric and martingale BMO spaces is well understood for doubling spaces, that is, when the measure of a ball in the given metric is comparable with the measure of its concentric dilations up to constants depending on the dilation factor but not on the chosen ball. Indeed, in this case the metric BMO is equivalent to a finite intersection of martingale BMO spaces constructed out of dyadic two-sided filtrations of atomic $\sigma$-algebras whose atoms look like balls; see [Conde 2013; Garnett and Jones 1982; Hytönen and Kairema 2012; Mei 2003]. What is more relevant, however, is that any of these martingale BMO spaces satisfies the following fundamental properties:

(i) Interpolation endpoint for the $L_{p}$ scale.

MSC2010: 42B20, 42B35, 46L52, $60 \mathrm{G} 46$.

Keywords: nondoubling measures, BMO spaces, interpolation, martingales, noncommutative harmonic analysis, classical harmonic analysis, Calderón-Zygmund theory. 
(ii) John-Nirenberg inequalities and $\mathrm{H}_{1}-\mathrm{BMO}$ duality.

(iii) $\mathrm{CZ}$ extrapolation: $L_{2}$-boundedness $\Rightarrow L_{\infty} \rightarrow \mathrm{BMO}$ boundedness.

Hence, these spaces yield at least as many endpoint estimates as the metric BMO.

The main goal of this paper is to construct BMO spaces satisfying the properties stated above for a larger class of measures, and to explore the implications of this construction to provide new endpoint estimates. The first attempts in this direction [Mateu et al. 2000; Nazarov et al. 2002] culminated in the work of Tolsa [2001] on so-called RBMO spaces. These spaces enjoy the above-mentioned properties for measures of polynomial growth. There are, however, a couple of open questions concerning Tolsa's construction. In the first place, Calderón-Zygmund extrapolation holds under a Lipschitz kernel condition instead of the more flexible Hörmander condition. Second, only interpolation of operators, has been studied but it seems to be unknown whether these spaces interpolate with the $L_{p}$ scale. These two problems were solved by Carbonaro, Mauceri and Meda [Carbonaro et al. 2009; 2010] for a different class of measures, based on similar results for the Gaussian measure on Euclidean spaces [Mauceri and Meda 2007]. The properties they imposed lead to locally doubling measures with certain concentration behavior at the boundary. In both cases - up to equivalence in the norm and additional conditions - only doubling balls are used to measure the mean oscillation of the function.

We present an alternative approach to these questions. Martingale BMO spaces always satisfy conditions (i) and (ii) above, with independence of the existence of a metric in the underlying measure space. The third property however requires additional structure on our BMO spaces. Indeed, assume for a moment that we work with a two-sided filtration $\left(\Sigma_{k}\right)_{k \in \mathbb{Z}}$ of atomic $\sigma$-subalgebras of $\Sigma$ with corresponding conditional expectations $\mathrm{E}_{\Sigma_{k}}$. If $\Pi$ denotes the union of atoms in our filtration, the corresponding martingale BMO norm is given by

$$
\|f\|_{\text {вмO }}=\sup _{k \in \mathbb{Z}}\left\|\mathrm{E}_{\Sigma_{k}}\left|f-\mathrm{E}_{\Sigma_{k-1}} f\right|^{2}\right\|_{\infty}^{1 / 2},
$$

which is larger than the function BMO norm

$$
\sup _{\mathrm{A} \in \Pi}\left(\frac{1}{\mu(\mathrm{A})} \int_{\mathrm{A}}\left|f(w)-\frac{1}{\mu(\mathrm{A})} \int_{\mathrm{A}} f d \mu\right|^{2} d \mu(w)\right)^{\frac{1}{2}} .
$$

Thus, if we admit from [Carbonaro et al. 2009; Tolsa 2001] that extrapolation for (nonlocal) CalderónZygmund operators imposes that our atoms be doubling - i.e., contained in a doubling ball of comparable measure or a union of at most $\mathrm{C}_{0}$ sets of this kind; see below - we immediately find obstructions to constructing filtrations satisfying this assumption for nondoubling spaces. We propose to consider a sort of intersection of two large BMO spaces as follows. Consider a $\sigma$-finite measure space $(\Omega, \Sigma, \mu)$ and two atomic $\sigma$-algebras $\Sigma_{\mathrm{a}}, \Sigma_{\mathrm{b}}$ of measurable sets in $\Sigma$ satisfying $\Sigma_{\mathrm{a}} \cap \Sigma_{\mathrm{b}}=\{\Omega, \varnothing\}$. Write $\mathrm{BMO}_{j}$ for any martingale BMO space over a filtration $\left(\Sigma_{j k}\right)_{k \geq 1}$ with $\Sigma_{j 1}=\Sigma_{j}$; then the seminorm

$$
\|f\|_{\mathrm{BMO}_{\Sigma_{\mathrm{ab}}}(\Omega)}=\max \left\{\left\|f-\mathrm{E}_{\Sigma_{\mathrm{a}}} f\right\|_{\mathrm{BMO}_{\mathrm{a}}},\left\|f-\mathrm{E}_{\Sigma_{\mathrm{b}}} f\right\|_{\mathrm{BMO}_{\mathrm{b}}}\right\}
$$


vanishes on constant functions precisely when $\Sigma_{\mathrm{a}} \cap \Sigma_{\mathrm{b}}$ is trivial. Let

$$
\mathrm{BMO}_{\Sigma_{\mathrm{ab}}}(\Omega)=\left\{f \in L_{\mathrm{loc}}^{1}(\Omega) \mid\|f\|_{\mathrm{BMO}_{\Sigma_{\mathrm{ab}}}(\Omega)}<\infty\right\} / \mathbb{C} .
$$

This settles a model of "large BMO spaces" which easily satisfy property (ii) and leave some room for property (iii). The problem reduces then to identify conditions on the pair $\left(\Sigma_{\mathrm{a}}, \Sigma_{\mathrm{b}}\right)$ so that BMO $\Sigma_{\mathrm{ab}}(\Omega)$ interpolates with the $L_{p}$ scale. A standard argument shows that this is the case when

$$
\|f\|_{L_{p}^{\circ}(\Omega)}:=\inf _{\mathrm{k} \in \mathbb{C}}\|f-\mathrm{k}\|_{p} \sim \max \left\{\left\|f-\mathrm{E}_{\Sigma_{\mathrm{a}}} f\right\|_{p},\left\|f-\mathrm{E}_{\Sigma_{\mathrm{b}}} f\right\|_{p}\right\}=:\|f\|_{L_{\Sigma_{\mathrm{ab}}}^{p}(\Omega)}
$$

for $2 \leq p<\infty$, where

$$
\begin{aligned}
L_{p}^{\circ}(\Omega) & =L_{p}(\Omega, \Sigma, \mu) / \mathbb{C} \\
L_{\Sigma_{\mathrm{ab}}}^{p}(\Omega) & =\left\{f \in L_{\mathrm{loc}}^{1}(\Omega) \mid\|f\|_{L_{\Sigma_{\mathrm{ab}}}^{p}(\Omega)}<\infty\right\} / \mathbb{C} \\
& =L_{p}(\Omega, \Sigma, \mu) / \Sigma_{\mathrm{a}} \wedge L_{p}(\Omega, \Sigma, \mu) / \Sigma_{\mathrm{b}} .
\end{aligned}
$$

Here, $L_{p}(\Omega, \Sigma, \mu) / \Sigma_{i}$ denotes the quotient space of $L_{p}(\Omega, \Sigma, \mu)$ by the subspace of $\Sigma_{i}$-measurable functions. More precisely, we have an isomorphism $L_{p}^{\circ}(\Omega) \simeq L_{\Sigma_{\mathrm{ab}}}^{p}(\Omega)$. It should be mentioned that this isomorphism fails in general, even for the Lebesgue measure in $\mathbb{R}^{n}$ and many "natural" choices of pairs $\left(\Sigma_{\mathrm{a}}, \Sigma_{\mathrm{b}}\right)$. Recall that $L_{p}^{\circ}(\Omega)=L_{p}(\Omega)$ for infinite measures. Note also that we use $\wedge$ and not $\cap$ since this space is not really an intersection; we shall also write $\mathrm{BMO}_{\Sigma_{\mathrm{ab}}}(\Omega)=\mathrm{BMO}_{\mathrm{a}}(\Omega) / \Sigma_{\mathrm{a}} \wedge \mathrm{BMO}_{\mathrm{b}}(\Omega) / \Sigma_{\mathrm{b}}$. To formulate a sufficient condition on $\left(\Sigma_{\mathrm{a}}, \Sigma_{\mathrm{b}}\right)$ for $L_{p}^{\circ}(\Omega) \simeq L_{\Sigma_{\mathrm{ab}}}^{p}(\Omega)$, let $\Pi_{j}$ be the set of atoms in $\Sigma_{j}$. When $\mu(\Omega)<\infty$ we shall consider two distinguished atoms $\left(A_{0}, B_{0}\right) \in \Pi_{\mathrm{a}} \times \Pi_{\mathrm{b}}$, while for $\mu$ not finite we take $A_{0}=B_{0}=\varnothing$ for notation consistency. Given $(A, B) \in \Pi_{\mathrm{a}} \times \Pi_{\mathrm{b}}$, set

$$
R_{A}=\left\{B^{\prime} \in \Pi_{\mathrm{b}} \mid \mu\left(A \cap B^{\prime}\right)>0\right\} \quad \text { and } \quad R_{B}=\left\{A^{\prime} \in \Pi_{\mathrm{a}} \mid \mu\left(A^{\prime} \cap B\right)>0\right\} .
$$

We will write $\left|R_{A}\right|$ and $\left|R_{B}\right|$ for the cardinality of these sets. The following is the main result of this paper, where we establish a condition on $\left(\Sigma_{\mathrm{a}}, \Sigma_{\mathrm{b}}\right)$ which suffices to make intersections and quotients commute in $L_{p}$ as described above. We will say that $\left(\Sigma_{\mathrm{a}}, \Sigma_{\mathrm{b}}\right)$ is an admissible covering of $(\Omega, \Sigma, \mu)$ when $\Sigma_{\mathrm{a}} \cap \Sigma_{\mathrm{b}}=\{\Omega, \varnothing\}$ and

$$
\min \left\{\sup _{A \in \Pi_{\mathrm{a}} \backslash\left\{A_{0}\right\}} \sum_{B \in R_{A}}\left|R_{B}\right| \frac{\mu(A \cap B)^{2}}{\mu(A) \mu(B)}, \sup _{B \in \Pi_{\mathrm{b}} \backslash\left\{B_{0}\right\}} \sum_{A \in R_{B}}\left|R_{A}\right| \frac{\mu(A \cap B)^{2}}{\mu(A) \mu(B)}\right\}<1 .
$$

Theorem A. Let $(\Omega, \Sigma, \mu)$ be a $\sigma$-finite measure space equipped with an admissible covering $\left(\Sigma_{\mathrm{a}}, \Sigma_{\mathrm{b}}\right)$. Then, for each $2 \leq p<\infty$, there exists a constant $c_{p}$, depending only on $p$ and the admissible covering, such that

$$
L_{p}^{\circ}(\Omega) \simeq{ }_{c_{p}} L_{\Sigma_{\mathrm{ab}}}^{p}(\Omega)
$$

Moreover, we have the desired complex interpolation result,

$$
\left[\mathrm{BMO}_{\Sigma_{\mathrm{ab}}}(\Omega), L_{1}^{\circ}(\Omega)\right]_{1 / q} \simeq_{c_{q}} L_{q}^{\circ}(\Omega) \quad(1<q<\infty),
$$

with $\mathrm{BMO}_{\Sigma_{\mathrm{ab}}}(\Omega)$ defined as above for any two martingale BMO spaces over $\left(\Sigma_{\mathrm{a}}, \Sigma_{\mathrm{b}}\right)$. 
The first assertion fails for $p=1, \infty$. On the other hand, both the John-Nirenberg inequalities and $\mathrm{H}_{1}-\mathrm{BMO}$ duality are easily formulated for these spaces. Therefore, we shall focus in what follows on condition (iii). Calderón-Zygmund extrapolation means that under a certain mild smoothness condition on the kernel, $L_{2}$-boundedness yields $L_{p}$-boundedness for $1<p<\infty$. As usual, we handle it by providing an endpoint estimate for interpolation. Let $d$ be a metric on $\Omega$ and denote by $\alpha \mathrm{B}$ the $\alpha$-dilation of a ball B. We impose the standard Hörmander kernel condition

$$
\sup _{\mathrm{B} d \text {-ball }} \sup _{z_{1}, z_{2} \in \mathrm{B}} \int_{\Omega \backslash \alpha \mathrm{B}}\left|k\left(z_{1}, x\right)-k\left(z_{2}, x\right)\right|+\left|k\left(x, z_{1}\right)-k\left(x, z_{2}\right)\right| d \mu(x)<\infty .
$$

Define a CZO on $(\Omega, \mu, d)$ as any linear map $T$ satisfying the following properties:

- $T$ is well-defined and bounded on $L_{2}(\Omega)$.

- The kernel representation for any $f \in \mathcal{C}_{c}(\Omega)$,

$$
T f(x)=\int_{\Omega} k(x, y) f(y) d \mu(y) \text { holds for } x \notin \operatorname{supp} f
$$

and some kernel $k: \Omega \times \Omega \backslash \Delta \rightarrow \mathbb{C}$ satisfying the Hörmander condition.

Given $\mathrm{C}_{0}>0$, a $\Sigma$-measurable set $A$ will be called $\left(\mathrm{C}_{0}, \alpha, \beta\right)$-doubling when it is the union of at most $\mathrm{C}_{0}$ sets which are contained in $(\alpha, \beta)$-doubling balls - balls $B$ such that $\mu(\alpha B) \leq \beta \mu(B)$ - of comparable measure up to the constant $\mathrm{C}_{0}$. Recall that a filtration $\left(\Sigma_{k}\right)_{k \geq 1}$ is called regular if $\mathrm{E}_{k} f \lesssim \mathrm{E}_{k-1} f$ for all $k>1$ and all $f \geq 0$.

Theorem B1. Let $\left(\Sigma_{\mathrm{a}}, \Sigma_{\mathrm{b}}\right)$ be an admissible covering of $(\Omega, \Sigma, \mu)$. Assume that $(\Omega, \Sigma, \mu)$ admits regular filtrations $\left(\Sigma_{j k}\right)_{k \geq 1}$ by successive refinement of $\Sigma_{j 1}=\Sigma_{j}$ for $j=\mathrm{a}, \mathrm{b}$ and that each atom in $\Sigma_{j k}$ is $\left(\mathrm{C}_{0}, \alpha, \beta\right)$-doubling for certain absolute constants $\mathrm{C}_{0}, \alpha, \beta>0$. Construct the spaces $\mathrm{BMO}_{\Sigma_{\mathrm{ab}}}(\Omega)$ which are defined over these filtrations. Then, every Calderón-Zygmund operator extends to a bounded map $L_{\infty}(\Omega) \rightarrow \mathrm{BMO}_{\Sigma_{\mathrm{ab}}}(\Omega)$, and $L_{p}(\Omega) \rightarrow L_{p}(\Omega)$ for $1<p<\infty$.

A few illustrations of Theorem B1 are the following:

- Doubling case: Theorem B1 recovers Calderón-Zygmund extrapolation on homogeneous spaces $(\Omega, \mu, d)$. We shall construct explicit pairs $\left(\Sigma_{\mathrm{a}}, \Sigma_{\mathrm{b}}\right)$ and martingale filtrations satisfying our assumptions.

- Polynomial growth: Given any $(\Omega, \mu, d)$ with polynomial growth, it is not difficult to construct atomic $\sigma$-algebras composed uniquely of doubling atoms, even giving admissible coverings. Under the existence of filtrations based on $\left(\Sigma_{\mathrm{a}}, \Sigma_{\mathrm{b}}\right)$ and composed of doubling atoms - regular or not - we may prove that Tolsa's RBMO sits inside our $\mathrm{BMO}_{\Sigma_{\mathrm{ab}}}(\Omega)$. This condition seems, unfortunately, a restrictive limit in Theorem B1. However, it can be checked in some concrete scenarios, like for

$$
d \mu(x)=\frac{d x}{1+|x|^{\beta}} \quad \text { with } \beta \gtrsim n^{3 / 2}
$$

in $\mathbb{R}^{n}$ equipped with the Euclidean metric. Note that $\mu$ is doubling for $\beta<n$. The key advantage over Tolsa's approach is that we only need to impose Hörmander kernel smoothness, instead of stronger Lipschitz conditions. This was also achieved by [Carbonaro et al. 2009; 2010] for another family of 
measures (see below) but not for the measures considered above, since they are drastically less concentrated at the boundary for any $\beta$.

- Concentration at the boundary: Carbonaro et al. [2009; 2010] proved that when $(\Omega, \mu, d)$ is locally doubling and the measure concentrates at the boundary of open sets in a certain sense - together with a purely metric condition that does not play any role here - a BMO space satisfying (i), (ii) and (iii) is possible. Their main examples in $\mathbb{R}^{n}$ with a weighted Euclidean metric were $d \mu(x)=e^{ \pm|x|^{\alpha}} d x$ and $\alpha>1$. The exponentially decreasing ones behave in some sense like the Gaussian measure, which was studied a few years before by Mauceri and Meda. It is of polynomial growth, so that the kernel smoothness condition was the main advantage with respect to Tolsa's approach. The exponentially increasing ones are not of polynomial growth. In this paper we shall remove their condition $\alpha>1$.

In the literature, we find other families of operators - with no need of a metric in the underlying space - which are close to CZOs in spirit. Martingale transforms are the simplest ones, but are local and much easier to bound. Nonlocal models include the so-called perfect dyadic CZOs and, most notably, Haar shift operators, which include prominent examples like the discrete Hilbert transform and dyadic paraproducts. In these cases, the Hörmander kernel condition can be replaced by

$$
\sup _{Q \text { dyadic cube } z_{1}, z_{2} \in Q} \sup _{\Omega \backslash \hat{Q}}\left|k\left(z_{1}, x\right)-k\left(z_{2}, x\right)\right|+\left|k\left(x, z_{1}\right)-k\left(x, z_{2}\right)\right| d \mu(x)<\infty,
$$

where $\hat{Q}$ denotes the dyadic father of $Q$. Our BMO spaces allow us to further replace dyadic cubes in dyadically doubling measure spaces — see [López-Sánchez et al. 2014] for recent progress on more general measures in this direction - by more general atoms. Namely, assume $\left(\Sigma_{\mathrm{a}}, \Sigma_{\mathrm{b}}\right)$ gives an admissible covering of $(\Omega, \Sigma, \mu)$. Consider regular filtrations of atomic $\sigma$-algebras $\left(\Sigma_{j k}\right)_{k \geq 1}$ with $\Sigma_{j 1}=\Sigma_{j}$ for $j=\mathrm{a}$, b. Let us write $\Pi_{j k}$ for the family of atoms in the atomic $\sigma$-algebra $\Sigma_{j k}$ and set $\Pi_{j}=\bigcup_{k \geq 1} \Pi_{j k}$. Then, consider the following Hörmander-type kernel condition, where the former role of the metric $d$ is replaced by the shape of our atoms in $\Pi=\Pi_{\mathrm{a}} \cup \Pi_{\mathrm{b}}$ :

$$
\sup _{A \in \Pi} \sup _{z_{1}, z_{2} \in A} \int_{\Omega \backslash \hat{A}}\left|k\left(z_{1}, x\right)-k\left(z_{2}, x\right)\right|+\left|k\left(x, z_{1}\right)-k\left(x, z_{2}\right)\right| d \mu(x)<\infty .
$$

Again, $\hat{A}$ denotes the minimal atom in the filtration of $A$ which contains $A$ properly, unless there is no such atom, in which case we pick $\hat{A}=A$. If we replace the Hörmander condition by this one, we obtain another class of "atomic" CZOs, which will be denoted in what follows by ACZO.

Theorem B2. Let $\left(\Sigma_{\mathrm{a}}, \Sigma_{\mathrm{b}}\right)$ be an admissible covering of $(\Omega, \Sigma, \mu)$. Assume in addition that $(\Omega, \Sigma, \mu)$ admits regular filtrations $\left(\Sigma_{j k}\right)_{k \geq 1}$ by successive refinement of $\Sigma_{j 1}=\Sigma_{j}$ for $j=\mathrm{a}$, b. Construct the spaces $\mathrm{BMO}_{\Sigma_{\mathrm{ab}}}(\Omega)$ which are defined over these filtrations. Then, every $\mathrm{ACZO}$ extends to a bounded map $L_{\infty}(\Omega) \rightarrow \mathrm{BMO}_{\Sigma_{\mathrm{ab}}}(\Omega)$, and $L_{p}(\Omega) \rightarrow L_{p}(\Omega)$ for $1<p<\infty$.

An advantage of Theorem B2 is that our kernel conditions are flexible, since we may carefully choose $\left(\Sigma_{a}, \Sigma_{b}\right)$ and the regular filtrations according to the concrete singular integral operator. It is worth mentioning that every $\sigma$-finite (atomless if $\mu$ is finite) measure space $(\Omega, \Sigma, \mu)$ has nontrivial admissible 
coverings. Of course, the regularity of the filtration is a light form of "doublingness" needed to emulate the classical argument in this setting. We will also provide weaker estimates for pseudolocal operators when the filtrations are not regular.

In contrast to [Carbonaro et al. 2009; 2010; Mauceri and Meda 2007; Tolsa 2001], our approach extends to matrix-valued functions, for which only limited results are known and no satisfactory nondoubling theory exists so far. In fact, this was our original motivation and the necessity of alternative arguments led to the results presented so far. We will postpone the discussion of the matrix-valued setting to the last section of this paper, which will allow those readers not familiar with noncommutative $L_{p}$ theory to isolate these results.

Our results above give some insight on the relation between nondoubling and martingale BMO theories; see [Conde-Alonso and Parcet 2014; Junge et al. $\geq 2015$ ] for other results along this line. In [CondeAlonso and Parcet 2014], we adapt Tolsa's ideas to give an atomic block description of martingale $\mathrm{H}_{1}$. Semigroup BMO spaces are used in [Junge et al. $\geq 2015$ ] to construct a Calderón-Zygmund theory that incorporates noncommutative measure spaces (von Neumann algebras) to the picture.

\section{Admissible coverings and BMO spaces}

In this section we recall some basic background around martingale BMO spaces and introduce our new class of BMO spaces. We will study standard properties of this class, like the existence of admissible coverings, John-Nirenberg inequalities and $\mathrm{H}_{1}-\mathrm{BMO}$ duality. The proof of Theorem A is more technical and will be postponed to Section 2 .

Martingale BMO spaces. Let $(\Omega, \Sigma, \mu)$ be a $\sigma$-finite measure space and consider a filtration $\left(\Sigma_{k}\right)_{k \geq 1}$ of $\Sigma$. In other words, we have $\Sigma_{k} \subset \Sigma_{k+1}$ and the union of the spaces $L_{\infty}\left(\Omega, \Sigma_{k}, \mu\right)$ is weak-* dense in $L_{\infty}(\Omega, \Sigma, \mu)$. Let $\mathrm{E}_{\Sigma_{k}}$ denote the conditional expectation onto $\Sigma_{k}$-measurable functions. Then, define the martingale BMO space associated to this filtration as the space of locally integrable functions $f: \Omega \rightarrow \mathbb{C}$ whose BMO norm,

$$
\|f\|_{\mathrm{BMO}}=\sup _{k \geq 1}\left\|\left(\mathrm{E}_{\Sigma_{k}}\left|f-\mathrm{E}_{\Sigma_{k-1}} f\right|^{2}\right)^{1} / 2\right\|_{\infty}
$$

is finite, where we use the convention $\mathrm{E}_{\Sigma_{0}} f=0$; see [Garsia 1973]. Another expression for the norm is

$$
\begin{aligned}
\|f\|_{\mathrm{BMO}} & =\sup _{k \geq 1}\left\|\left|d f_{k}\right|^{2}+\sum_{n>k} \mathrm{E}_{\Sigma_{k}}\left|d f_{n}\right|^{2}\right\|_{\infty}^{\frac{1}{2}} \\
& \sim\left[\sup _{k \geq 1}\left\|\left(\mathrm{E}_{\Sigma_{k}}\left|f-\mathrm{E}_{\Sigma_{k}} f\right|^{2}\right)^{1} / 2\right\|_{\infty}+\left\|\mathrm{E}_{\Sigma_{1}} f\right\|_{\infty}\right]+\sup _{k>1}\left\|d f_{k}\right\|_{\infty},
\end{aligned}
$$

where $d f_{k}=\Delta_{k} f=\mathrm{E}_{\Sigma_{k}} f-\mathrm{E}_{\Sigma_{k-1}} f$. According to [Janson and Jones 1982], [BMO, $\left.L_{1}(\Omega)\right]_{1 / p} \simeq L_{p}(\Omega)$ for any filtration we pick. The bracketed term in the right-hand side above is called the martingale bmo norm of $f$, and it is closer to the standard expressions to measure the mean oscillation of a function. Namely, if the $\sigma$-algebras $\Sigma_{k}$ are atomic and if $\Pi_{k}$ denotes the atoms in $\Sigma_{k}$ and $\Pi=\bigcup_{k \geq 1} \Pi_{k}$, we deduce 
that

$$
\begin{aligned}
\|f\|_{\text {bmo }} & =\sup _{k \geq 1}\left\|\left(\mathrm{E}_{\Sigma_{k}}\left|f-\mathrm{E}_{\Sigma_{k}} f\right|^{2}\right)^{1} / 2\right\|_{\infty}+\left\|\mathrm{E}_{\Sigma_{1}} f\right\|_{\infty} \\
& =\sup _{A \in \Pi}\left(\frac{1}{\mu(A)} \int_{A}\left|f(w)-\frac{1}{\mu(A)} \int_{A} f d \mu\right|^{2} d \mu(w)\right)^{\frac{1}{2}}+\sup _{A \in \Pi_{1}}\left|\frac{1}{\mu(A)} \int_{A} f d \mu\right| .
\end{aligned}
$$

Of course, using a selected family of atoms makes $L_{p}$-interpolation fail in general for bmo. The extra term in BMO corrects this. This should be compared with the extra condition in the definition of Tolsa's RBMO. On the other hand, bmo spaces have good interpolation properties with little Hardy spaces $\mathrm{h}_{p}$. Namely, according to [Bekjan et al. 2010] we have $\left[\mathrm{bmo}, \mathrm{h}_{1}\right]_{1 / p} \simeq \mathrm{h}_{p}$ for any filtration, where $\mathrm{h}_{p}$ is the closure of the space of finite martingales in $L_{p}$ with respect to the norm

$$
\|f\|_{\mathrm{h}_{p}}=\left\|\left(\sum_{k \geq 1} \mathrm{E}_{\Sigma_{k-1}}\left|d f_{k}\right|^{2}\right)^{\frac{1}{2}}\right\|_{p} ;
$$

this time the convention is $\mathrm{E}_{\Sigma_{0}}\left|d f_{1}\right|^{2}=\left|\mathrm{E}_{\Sigma_{1}} f\right|^{2}$. In contrast to other BMO spaces seminorms, paradoxically, we will need to quotient out certain spaces. Note that, for $\Sigma_{1}$-measurable functions, the norms above coincide with the $L_{\infty}$ norm

$$
\left\|\mathrm{E}_{\Sigma_{1}} f\right\|_{\mathrm{BMO}}=\left\|\mathrm{E}_{\Sigma_{1}} f\right\|_{\mathrm{bmo}}=\left\|\mathrm{E}_{\Sigma_{1}} f\right\|_{L_{\infty}(\Omega)} .
$$

If we define the seminorms

$$
\begin{aligned}
\|f\|_{\mathrm{bmo}}^{\circ} & =\left\|f-\mathrm{E}_{\Sigma_{1}} f\right\|_{\mathrm{bmo}}, \\
\|f\|_{\mathrm{BMO}}^{\circ} & =\left\|f-\mathrm{E}_{\Sigma_{1}} f\right\|_{\mathrm{BMO}},
\end{aligned}
$$

we obtain complemented subspaces $\mathrm{BMO}_{\Sigma_{1}}=J_{\Sigma_{1}}$ (BMO) using the projection $J_{\Sigma_{1}}=\mathrm{id}-\mathrm{E}_{\Sigma_{1}}$. Indeed, it is a simple exercise using Jensen's conditional inequality $\left|\mathrm{E}_{\Sigma_{1}} f\right|^{2} \leq \mathrm{E}_{\Sigma_{1}}|f|^{2}$; details are left to the reader. Since $J_{\Sigma_{1}}$ is also bounded on $\mathrm{h}_{p}$ and $L_{p}$, the previous interpolation results imply the following isomorphisms for $1<p<\infty$ :

$$
\begin{aligned}
{\left[J_{\Sigma_{1}}(\mathrm{bmo}), J_{\Sigma_{1}}\left(\mathrm{~h}_{1}(\Omega)\right)\right]_{1 / p} } & \simeq J_{\Sigma_{1}}\left(\mathrm{~h}_{p}(\Omega)\right), \\
{\left[J_{\Sigma_{1}}(\mathrm{BMO}), J_{\Sigma_{1}}\left(L_{1}(\Omega)\right)\right]_{1 / p} } & \simeq J_{\Sigma_{1}}\left(L_{p}(\Omega)\right) .
\end{aligned}
$$

Note that $J_{\Sigma_{1}}\left(L_{p}(\Omega)\right) \simeq L_{p}(\Omega, \Sigma, \mu) / \Sigma_{1}$ in the terminology of the introduction.

Remark 1.1. It is worth mentioning that the Janson-Jones interpolation theorem [1982] holds for arbitrary filtrations. In particular, we could replace $\left(\Sigma_{k}\right)_{k \geq 1}$ by $\left(\Sigma_{k}\right)_{k \geq \mathrm{N}}$ for some large N, and the latter BMO comes equipped with the norm

$$
\sup _{k \geq \mathrm{N}}\left\|\left(\mathrm{E}_{\Sigma_{k}}\left|f-\mathrm{E}_{\Sigma_{k}} f\right|^{2}\right)^{1} / 2\right\|_{\infty}+\left\|\mathrm{E}_{\Sigma_{\mathrm{N}}} f\right\|_{\infty}+\sup _{k>\mathrm{N}}\left\|d f_{k}\right\|_{\infty}
$$

When $\mathrm{N}$ is large enough, the middle term dominates the others and we get spaces which are closer and closer to $L_{\infty}(\Omega)$. In contrast, when we quotient out the first $\sigma$-algebra by using the $J$-projections, 
it follows from the interpolation identities above that the starting $\sigma$-algebra significantly affects the interpolated space. This justifies, in part, our need to intersect two such spaces in this paper.

BMO spaces for admissible coverings. Let $(\Omega, \Sigma, \mu)$ be a $\sigma$-finite measure space and consider two atomic $\sigma$-algebras $\Sigma_{\mathrm{a}}, \Sigma_{\mathrm{b}}$ of measurable sets in $\Sigma$. Let $\Pi_{j}$ be the set of atoms in $\Sigma_{j}$ for $j=\mathrm{a}$, b. When $\mu(\Omega)<\infty$, we shall consider two distinguished atoms $\left(A_{0}, B_{0}\right) \in \Pi_{\mathrm{a}} \times \Pi_{\mathrm{b}}$. If $\mu$ is not finite, take $A_{0}=B_{0}=\varnothing$. Given $A \in \Pi_{\mathrm{a}}$, set

$$
R_{A}=\left\{B^{\prime} \in \Pi_{\mathrm{b}} \mid \mu\left(A \cap B^{\prime}\right)>0\right\} .
$$

Define $R_{B}$ for $B \in \Pi_{\mathrm{b}}$ similarly. The pair $\left(\Sigma_{\mathrm{a}}, \Sigma_{\mathrm{b}}\right)$ is called an admissible covering of $(\Omega, \Sigma, \mu)$ when $\Sigma_{\mathrm{a}} \cap \Sigma_{\mathrm{b}}=\{\Omega, \varnothing\}$ and

$$
\min \left\{\sup _{A \in \Pi_{\mathrm{a}} \backslash\left\{A_{0}\right\}} \sum_{B \in R_{A}}\left|R_{B}\right| \frac{\mu(A \cap B)^{2}}{\mu(A) \mu(B)}, \sup _{B \in \Pi_{\mathrm{b}} \backslash\left\{B_{0}\right\}} \sum_{A \in R_{B}}\left|R_{A}\right| \frac{\mu(A \cap B)^{2}}{\mu(A) \mu(B)}\right\}<1 .
$$

One can view the condition above as a weak version of the concentration of measure near the boundary that appeared in [Carbonaro et al. 2009]. In particular, it is not a geometric notion, but only a measure-theoretic one (see Remark 3.3 for more details). Now, consider any pair of filtrations $\left(\Sigma_{j k}\right)_{k \geq 1}$ with $\Sigma_{j 1}=\Sigma_{j}$ for $j=\mathrm{a}, \mathrm{b}$, and construct the corresponding martingale $\mathrm{BMO}$ spaces $\mathrm{BMO}_{\mathrm{a}}$ and $\mathrm{BMO}_{\mathrm{b}}$. As in the previous subsection, we quotient out the $\Sigma_{j}$-measurable functions and set, as we did in the introduction,

$$
\begin{aligned}
\mathrm{BMO}_{\Sigma_{j}}(\Omega) & =J_{\Sigma_{j}}\left(\mathrm{BMO}_{j}\right), \\
\mathrm{BMO}_{\Sigma_{\mathrm{ab}}}(\Omega) & =\mathrm{BMO}_{\Sigma_{\mathrm{a}}}(\Omega) \wedge \mathrm{BMO}_{\Sigma_{\mathrm{b}}}(\Omega)=\left\{f \in L_{\mathrm{loc}}^{1}(\Omega) \mid\|f\|_{\mathrm{BMO}_{\Sigma_{\mathrm{ab}}}(\Omega)}<\infty\right\} / \mathbb{C}
\end{aligned}
$$

In the following, we construct admissible coverings for $\sigma$-finite measure spaces. The procedure we employ is quite general. In concrete scenarios, other admissible coverings can be constructed enjoying additional properties as required in Theorems B1 and B2; these examples will be given later in this paper.

Remark 1.2. The classical BMO on Euclidean spaces can be decomposed as an intersection of finitely many martingale BMO spaces, the number of which depends on the dimension [Conde 2013; Garnett and Jones 1982; Mei 2003]. In contrast, we just consider "intersections" of two martingale BMOs. Note this makes our spaces larger and still amenable for interpolation, which gives some extra room to obtain endpoint estimates for singular integral operators. The main reason why this is possible is that our approach just relies on measure-theoretic properties and does not rely on the geometry of the underlying space, as will become clear in the sequel.

Lemma 1.3. Let $(\Omega, \Sigma, \mu)$ be a $\sigma$-finite measure space. Then:

(i) If $\mu(\Omega)=\infty$, it admits an admissible covering.

(ii) If $\mu(\Omega)<\infty$ and $\mu$ is atomless, it admits an admissible covering.

Proof. If $\mu(\Omega)=\infty$, pick $A_{0}=\tilde{A}_{0}=B_{0}=\tilde{B}_{0}=\varnothing$,

$$
A_{j}=\tilde{A}_{j} \backslash \tilde{A}_{j-1} \quad \text { and } \quad B_{j}=\tilde{B}_{j} \backslash \tilde{B}_{j-1},
$$


where $\varnothing \neq \tilde{A}_{1} \varsubsetneqq \tilde{B}_{1} \varsubsetneqq \tilde{A}_{2} \varsubsetneqq \tilde{B}_{2} \varsubsetneqq \tilde{A}_{3} \varsubsetneqq \cdots$ are $\Sigma$-measurable sets chosen so that

$$
\min \left\{\frac{\mu\left(\tilde{B}_{j} \backslash \tilde{B}_{j-1}\right)}{\mu\left(\tilde{A}_{j}\right)}, \frac{\mu\left(\tilde{A}_{j+1} \backslash \tilde{A}_{j}\right)}{\mu\left(\tilde{B}_{j}\right)}\right\}>\lambda>4 \text { for all } j \geq 1 .
$$

It is at this point that we have used that $\mu(\Omega)=\infty$. Let $\Sigma_{\mathrm{a}}$ be the atomic $\sigma$-algebra generated by the atoms $\left(A_{j}\right)_{j \geq 1}$. Similarly, define $\Sigma_{\mathrm{b}}=\sigma\left\langle B_{j}: j \geq 1\right\rangle$. It is clear by construction that

$$
\Sigma_{\mathrm{a}} \cap \Sigma_{\mathrm{b}}=\{\Omega, \varnothing\} .
$$

On the other hand, $\left|R_{B}\right|=2$ for every atom $B$ in $\Sigma_{\mathrm{b}}$. Therefore, it remains to show that

$$
\sup _{j \geq 1}\left[\frac{\mu\left(A_{j} \cap B_{j-1}\right)^{2}}{\mu\left(A_{j}\right) \mu\left(B_{j-1}\right)}+\frac{\mu\left(A_{j} \cap B_{j}\right)^{2}}{\mu\left(A_{j}\right) \mu\left(B_{j}\right)}\right]<\frac{1}{2} .
$$

Note that the first summand above vanishes for $j=1$. The rest of terms are smaller than $1 / \lambda$, according to our conditions, so that $\lambda>4$ suffices. When $\mu(\Omega)<\infty$ we may assume that $\mu(\Omega)=1$, since renormalization does not affect our definition of admissible covering. We use again a "corona-type partition"

$$
\varnothing \neq \tilde{A}_{0} \varsubsetneqq \tilde{B}_{0} \varsubsetneqq \tilde{A}_{1} \varsubsetneqq \tilde{B}_{1} \varsubsetneqq \tilde{A}_{2} \varsubsetneqq \cdots
$$

satisfying $\mu\left(\tilde{A}_{0}\right)=1-\zeta, \mu\left(\tilde{B}_{0} \backslash \tilde{A}_{0}\right)=\zeta(1-\zeta)$ and the relations

$$
\mu\left(\tilde{A}_{j+1} \backslash \tilde{B}_{j}\right)=\zeta \mu\left(\tilde{B}_{j} \backslash \tilde{A}_{j}\right) \quad \text { and } \quad \mu\left(\tilde{B}_{j+1} \backslash \tilde{A}_{j+1}\right)=\zeta \mu\left(\tilde{A}_{j+1} \backslash \tilde{B}_{j}\right) \quad \text { for } j \geq 0 .
$$

This is where we use the fact that $\mu$ has no atoms. Define $A_{0}=\tilde{A}_{0}, B_{0}=\tilde{B}_{0}, A_{j}=\tilde{A}_{j} \backslash \tilde{A}_{j-1}$ and $B_{j}=\tilde{B}_{j} \backslash \tilde{B}_{j-1}$ for $j \geq 1$. The $\sigma$-algebras $\Sigma_{\mathrm{a}}$ and $\Sigma_{\mathrm{b}}$ are the ones generated by $\left(A_{j}\right)_{j \geq 0}$ and $\left(B_{j}\right)_{j \geq 0}$, respectively. In order to show that $\Omega=\bigcup_{j \geq 0} A_{j}=\bigcup_{j \geq 0} B_{j}$, let us prove that we have

$$
\sum_{j \geq 0} \mu\left(A_{j}\right)=\sum_{j \geq 0} \mu\left(B_{j}\right)=1 .
$$

Indeed, if $j \geq 2$ we have

$$
\begin{aligned}
\mu\left(A_{j}\right) & =\mu\left(\tilde{A}_{j} \backslash \tilde{A}_{j-1}\right) \\
& =(1+\zeta) \mu\left(\tilde{B}_{j-1} \backslash \tilde{A}_{j-1}\right)=\zeta(1+\zeta) \mu\left(\tilde{A}_{j-1} \backslash \tilde{B}_{j-2}\right) \\
& =\zeta(1+\zeta)\left[\mu\left(\tilde{A}_{j-1} \backslash \tilde{A}_{j-2}\right)-\frac{1}{\zeta} \mu\left(\tilde{A}_{j-1} \backslash \tilde{B}_{j-2}\right)\right]=\zeta^{2} \mu\left(A_{j-1}\right)
\end{aligned}
$$

Therefore, since $\mu\left(A_{0}\right)=1-\zeta$ and $\mu\left(A_{1}\right)=\zeta\left(1-\zeta^{2}\right)$, we deduce immediately that $\sum_{j \geq 0} \mu\left(A_{j}\right)=1$. The sum $\sum_{j} \mu\left(B_{j}\right)$ also equals 1 since the two families are nested. The condition $\Sigma_{\mathrm{a}} \cap \Sigma_{\mathrm{b}}=\varnothing$ follows again by construction. Finally, since $\left|R_{B}\right|=2$ for all atoms $B=B_{j}$, it suffices one more time to prove that

$$
\sup _{j \geq 1}\left[\frac{\mu\left(A_{j} \cap B_{j-1}\right)^{2}}{\mu\left(A_{j}\right) \mu\left(B_{j-1}\right)}+\frac{\mu\left(A_{j} \cap B_{j}\right)^{2}}{\mu\left(A_{j}\right) \mu\left(B_{j}\right)}\right]<\frac{1}{2} .
$$


According to our construction, the left-hand side can be majorized by

$$
\frac{\mu\left(A_{j} \cap B_{j-1}\right)^{2}}{\mu\left(A_{j}\right) \mu\left(B_{j-1}\right)}+\frac{\mu\left(A_{j} \cap B_{j}\right)^{2}}{\mu\left(A_{j}\right) \mu\left(B_{j}\right)} \leq \frac{\mu\left(\tilde{B}_{j-1} \backslash \tilde{A}_{j-1}\right)}{\mu\left(B_{j-1}\right)}+\frac{\mu\left(\tilde{A}_{j} \backslash \tilde{B}_{j-1}\right)}{\mu\left(A_{j}\right)} .
$$

On the other hand, arguing as before, we may obtain the identities

$$
\begin{aligned}
\mu\left(A_{j}\right) & =\zeta^{2(j-1)}\left(\zeta-\zeta^{3}\right), \quad \mu\left(\tilde{A}_{j} \backslash \tilde{B}_{j-1}\right)=\zeta^{2 j}(1-\zeta), \\
\mu\left(B_{j-1}\right) & =\zeta^{2(j-1)}\left(1-\zeta^{2}\right), \quad \mu\left(\tilde{B}_{j-1} \backslash \tilde{A}_{j-1}\right)=\zeta^{2(j-1)}\left(\zeta-\zeta^{2}\right) .
\end{aligned}
$$

This gives a bound $2 \zeta /(1+\zeta)$. It suffices for $\zeta<\frac{1}{3}$. The proof is complete.

Remark 1.4. All fully supported probability measures on $\mathbb{R}^{n}$ are nondoubling. In fact, this also holds for probability measures supported on unbounded sets. In particular, we hope Lemma 1.3 together with Theorems B1 and B2 might open a door to further insight into Calderón-Zygmund theory for these measures.

John-Nirenberg inequalities, atomic $\mathbf{H}_{1}$ and duality. We now transfer some well-known properties of martingale BMO spaces to our new class of spaces. The analogue of John-Nirenberg inequalities [1961] for martingale $\mathrm{BMO}$ spaces can be stated as follows:

$$
\sup _{k \geq 1} \sup _{A \in \Sigma_{k}} \frac{1}{\mu(A)} \mu\left(A \cap\left\{\left|f-\mathrm{E}_{\Sigma_{k-1}} f\right|>\lambda\right\}\right) \lesssim \exp \left(-\frac{c \lambda}{\|f\|_{\text {BMO }}}\right) \text { for all } \lambda>0,
$$

where the martingale BMO is constructed over the filtration $\left(\Sigma_{k}\right)_{k \geq 1}$ and we use the convention $\mathrm{E}_{\Sigma_{0}} f=0$. The proof can be found in [Garsia 1973]. An important consequence of this inequality is the $p$-invariance of the BMO norm. To be more precise, the martingale BMO norm admits the equivalent expressions, for any $0<p<\infty$,

$$
\|f\|_{\mathrm{BMO}} \sim \sup _{k \geq 1}\left\|\left(\mathrm{E}_{\Sigma_{k}}\left|f-\mathrm{E}_{\Sigma_{k-1}} f\right|^{p}\right)^{1 / p}\right\|_{\infty} .
$$

If we replace $f$ by $J_{\Sigma_{1}} f=f-\mathrm{E}_{\Sigma_{1}} f$ in both inequalities, we immediately obtain the corresponding analogues for the BMO spaces which quotient out $\Sigma_{1}$-measurable functions, introduced above. Namely, the only difference is that we should read John-Nirenberg inequalities under the convention that $\mathrm{E}_{\Sigma_{0}} f=\mathrm{E}_{\Sigma_{1}} f$, and the BMO norm is given by $\|\cdot\|_{\mathrm{BMO}}^{\circ}$ instead. If we intersect two of these BMO spaces, we get JohnNirenberg-type inequalities for our spaces $\mathrm{BMO}_{\Sigma_{\mathrm{ab}}}(\Omega)$ associated to an admissible covering $\left(\Sigma_{\mathrm{a}}, \Sigma_{\mathrm{b}}\right)$ by taking again $\mathrm{E}_{\Sigma_{0}} f=\mathrm{E}_{\Sigma_{1}} f$ :

$$
\begin{gathered}
\|f\|_{\mathrm{BMO}_{\Sigma_{\mathrm{ab}}}(\Omega)} \sim \max _{j=\mathrm{a}, \mathrm{b}} \sup _{k \geq 1}\left\|\left(\mathrm{E}_{\Sigma_{j k}}\left|f-\mathrm{E}_{\Sigma_{j(k-1)}} f\right|^{p}\right)^{1 / p}\right\|_{\infty}, \\
\sup _{j=\mathrm{a}, \mathrm{b}} \sup _{\substack{k \geq 1 \\
A \in \Sigma_{j k}}} \frac{1}{\mu(A)} \mu\left(A \cap\left\{\left|f-\mathrm{E}_{\Sigma_{j(k-1)}} f\right|>\lambda\right\}\right) \lesssim \exp \left(-\frac{c \lambda}{\|f\|_{\mathrm{BMO}_{\Sigma_{\mathrm{ab}}}(\Omega)}}\right) .
\end{gathered}
$$

Let us now consider $\mathrm{H}_{1}-\mathrm{BMO}$ duality in our context. In the literature we find several equivalent descriptions of martingale $\mathrm{H}_{1}$ spaces, via Doob's maximal function, martingale square function or 
conditional square function. Namely, $\mathrm{H}_{1}$ can be defined as the closure of the space of finite $L_{1}$ martingales with respect to any of the norms

$$
\left\|\sup _{k \geq 1}\left|\mathrm{E}_{\Sigma_{k}} f\right|\right\|_{1} \sim\left\|\left(\sum_{k \geq 1}\left|d f_{k}\right|^{2}\right)^{\frac{1}{2}}\right\|_{1} \sim \sum_{k \geq 1}\left\|d f_{k}\right\|_{1}+\left\|\left(\sum_{k \geq 1} \mathrm{E}_{\Sigma_{k-1}}\left|d f_{k}\right|^{2}\right)^{\frac{1}{2}}\right\|_{1} .
$$

We refer to [Davis 1970] for the equivalences above and to [Garsia 1973] for the duality theorem, which claims that $\mathrm{H}_{1}^{*} \simeq \mathrm{BMO}$, a martingale analogue of the Fefferman-Stein duality theorem. Let us now consider atomic descriptions of these spaces. The term "atom" unfortunately appears here in several settings - $\sigma$-algebras, measures and Hardy spaces - with different meanings, but it will be clear which one is used from the context. Atomic descriptions are not possible for arbitrary $\mathrm{H}_{1}$ - see [Conde-Alonso and Parcet 2014] for an "atomic block" description both in the commutative and noncommutative settings but there are such results for $\mathrm{h}_{1}$ (defined above). A $\Sigma$-measurable function $a \in L_{2}(\Omega)$ is called an atom when there exists $k \geq 1$ and $A \in \Sigma_{k}$ with

$$
\operatorname{supp}(a) \subset A, \quad \mathrm{E}_{\Sigma_{k}}(a)=0, \quad\|a\|_{2} \leq \mu(A)^{-1 / 2} .
$$

The atomic $\mathrm{h}_{1}$ is defined as the space of functions of the form $f=\sum_{j} \lambda_{j} a_{j}$ with the $a_{j}$ atoms. The norm is the infimum of $\sum_{j}\left|\lambda_{j}\right|$ over all such possible expressions for the function $f$. This space is isomorphic to $h_{1}$; see [Garsia 1973]. In particular, it is also isomorphic to $\mathrm{H}_{1}$ when the filtration is regular. This will be enough for our purposes, since we will only use $\mathrm{H}_{1}-\mathrm{BMO}$ duality for regular filtrations. Now, given two filtrations $\left(\Sigma_{j k}\right)_{k \geq 1}$ with $\Sigma_{j 1}=\Sigma_{j}$ for $j=\mathrm{a}$, b, let $\mathrm{H}_{1 j}$ be the corresponding $\mathrm{H}_{1}$ spaces. Define

$$
\mathrm{H}_{\Sigma_{\mathrm{ab}}}^{1}(\Omega)=\left\{f \in L_{1}(\Omega) \mid\|f\|_{\mathrm{H}_{1}}=\inf _{\substack{f=f_{1}+f_{2} \\ \mathrm{E}_{\Sigma_{\mathrm{a}}} f_{1}=\mathrm{E}_{\Sigma_{\mathrm{b}}} f_{2}=0}}\left\|f_{1}\right\|_{\mathrm{H}_{1 \mathrm{a}}}+\left\|f_{2}\right\|_{\mathrm{H}_{1 \mathrm{~b}}}<\infty\right\} .
$$

Then, all the results above apply. In particular, we have

$$
\mathrm{H}_{\Sigma_{\mathrm{ab}}}^{1}(\Omega)^{*} \simeq \mathrm{BMO}_{\Sigma_{\mathrm{ab}}}(\Omega)
$$

\section{Interpolation: proof of Theorem A}

Proof of Theorem A. The argument is a bit lengthy, so we have divided it into several steps. We will assume that $\mu$ is a finite measure on $\Omega$ - normalized so that $\mu(\Omega)=1$ - since this case is more technical. The slight modifications needed for the nonfinite case will be explained in the last step of the proof.

Step 1: Intersection of quotients. Let us first show that the interpolation result follows from the first assertion of Theorem A. Namely, given an admissible covering $\left(\Sigma_{\mathrm{a}}, \Sigma_{\mathrm{b}}\right)$ of $(\Omega, \Sigma, \mu)$ and filtrations $\left(\Sigma_{j k}\right)_{k \geq 1}$ with $\Sigma_{j 1}=\Sigma_{j}$ for $j=\mathrm{a}$, b, let $\mathrm{BMO}_{j}$ be the corresponding martingale BMO spaces. It is clear that

$$
\begin{aligned}
\|f\|_{L_{q}^{\circ}(\Omega)}=\|f\|_{\left[L_{\infty}^{\circ}(\Omega), L_{1}^{\circ}(\Omega)\right]_{1 / q}} & \geq\|f\|_{\left[\mathrm{BMO}_{\Sigma_{\mathrm{ab}}}(\Omega), L_{1}^{\circ}(\Omega)\right]_{1 / q}} \\
& \geq \max _{j=\mathrm{a}, \mathrm{b}}\left\|f-\mathrm{E}_{\Sigma_{j}} f\right\|_{\left[J_{\Sigma_{j}}\left(\mathrm{BMO}_{j}\right), J_{\Sigma_{j}}\left(L_{1}(\Omega)\right)\right]_{1 / q}} \\
& \simeq \max _{j=\mathrm{a}, \mathrm{b}}\left\|f-\mathrm{E}_{\Sigma_{j}} f\right\|_{L_{\Sigma_{\mathrm{j}}}^{q}(\Omega)}=\|f\|_{L_{\Sigma_{\mathrm{ab}}}^{q}(\Omega) .}
\end{aligned}
$$


For $q \geq 2$, this implies

$$
L_{q}^{\circ}(\Omega) \subset\left[\mathrm{BMO}_{\Sigma_{\mathrm{ab}}}(\Omega), L_{1}^{\circ}(\Omega)\right]_{1 / q} \subset L_{\Sigma_{\mathrm{ab}}}^{q}(\Omega) .
$$

Thus, the result follows from the isomorphism $L_{q}^{\circ}(\Omega) \simeq L_{\Sigma_{\mathrm{ab}}}^{q}(\Omega)$. The interpolation result for $1<q<2$ follows from this and the well-known reiteration theorem [Bergh and Löfström 1976].

Step 2: Reduction to strict contractions. The rest of the proof will be devoted to justify the first assertion of Theorem A. We claim that such an isomorphism holds whenever we can find a constant $0<c_{p}\left(\Sigma_{\mathrm{ab}}\right)<1$ such that, for every mean-zero function $f \in L_{p}(\Omega)$,

$$
\min \left\{\left\|\mathrm{E}_{\Sigma_{\mathrm{a}}} \mathrm{E}_{\Sigma_{\mathrm{b}}} f\right\|_{p},\left\|\mathrm{E}_{\Sigma_{\mathrm{b}}} \mathrm{E}_{\Sigma_{\mathrm{a}}} f\right\|_{p}\right\} \leq c_{p}\left(\Sigma_{\mathrm{ab}}\right)\|f\|_{p} .
$$

Indeed, if $\mathrm{E} \phi=\int_{\Omega} \phi d \mu$, we first observe that

$$
\begin{aligned}
\|\phi\|_{L_{p}^{\circ}(\Omega)} & \sim\|\phi-\mathrm{E} \phi\|_{p} \sim \inf _{\mathrm{k} \in \mathbb{C}}\|\phi-\mathrm{k}\|_{p}, \\
\|\phi\|_{J_{\Sigma_{j}}\left(L_{p}(\Omega)\right)} & \sim\left\|\phi-\mathrm{E}_{\Sigma_{j}} \phi\right\|_{p} \sim \inf _{\varphi \Sigma_{j} \text {-measurable }}\|\phi-\varphi\|_{p} .
\end{aligned}
$$

Therefore, our goal in what follows is to show that

$$
\|\phi-\mathrm{E} \phi\|_{p} \sim\left\|\phi-\mathrm{E}_{\Sigma_{\mathrm{a}}} \phi\right\|_{p}+\left\|\phi-\mathrm{E}_{\Sigma_{\mathrm{b}}} \phi\right\|_{p} \quad \text { for every } \phi \in L_{p}(\Omega) .
$$

The lower estimate is trivial. For the upper estimate, we shall use (2-1). Assume that the minimum above is attained at the first term (say) and let $f=\phi-\mathrm{E} \phi$ be a mean-zero function. We then find

$$
\left\|\mathrm{E}_{\Sigma_{\mathrm{a}}} \mathrm{E}_{\Sigma_{\mathrm{b}}} f\right\|_{p} \leq c_{p}\left(\Sigma_{\mathrm{ab}}\right)\|f\|_{p} \leq c_{p}\left(\Sigma_{\mathrm{ab}}\right)\left[\left\|f-\mathrm{E}_{\Sigma_{\mathrm{a}}} f\right\|_{p}+\left\|\mathrm{E}_{\Sigma_{\mathrm{a}}}\left(f-\mathrm{E}_{\Sigma_{\mathrm{b}}} f\right)\right\|_{p}+\left\|\mathrm{E}_{\Sigma_{\mathrm{a}}} \mathrm{E}_{\Sigma_{\mathrm{b}}} f\right\|_{p}\right],
$$

which implies

$$
\left\|\mathrm{E}_{\Sigma_{\mathrm{a}}} \mathrm{E}_{\Sigma_{\mathrm{b}}} f\right\|_{p} \leq \frac{c_{p}\left(\Sigma_{\mathrm{ab}}\right)}{1-c_{p}\left(\Sigma_{\mathrm{ab}}\right)}\left[\left\|\phi-\mathrm{E}_{\Sigma_{\mathrm{a}}} \phi\right\|_{p}+\left\|\phi-\mathrm{E}_{\Sigma_{\mathrm{b}}} \phi\right\|_{p}\right] .
$$

This inequality is all we need, since the upper estimate follows from it:

$$
\begin{aligned}
\|\phi-\mathrm{E} \phi\|_{p} \leq\left\|\mathrm{E}_{\Sigma_{\mathrm{a}}} \phi-\mathrm{E} \phi\right\|_{p}+\left\|\phi-\mathrm{E}_{\Sigma_{\mathrm{a}}} \phi\right\|_{p} & \leq\left\|\mathrm{E}_{\Sigma_{\mathrm{a}}} \mathrm{E}_{\Sigma_{\mathrm{b}}} f\right\|_{p}+\left\|\mathrm{E}_{\Sigma_{\mathrm{a}}}\left(\phi-\mathrm{E}_{\Sigma_{\mathrm{b}}} \phi\right)\right\|_{p}+\left\|\phi-\mathrm{E}_{\Sigma_{\mathrm{a}}} \phi\right\|_{p} \\
& \leq \frac{1}{1-c_{p}\left(\Sigma_{\mathrm{ab}}\right)}\left[\left\|\phi-\mathrm{E}_{\Sigma_{\mathrm{a}}} \phi\right\|_{p}+\left\|\phi-\mathrm{E}_{\Sigma_{\mathrm{b}}} \phi\right\|_{p}\right] .
\end{aligned}
$$

Step 3: The case $\boldsymbol{p}=2$. Recall that we are assuming for the moment that $\mu(\Omega)=1$, and in that case we may consider two distinguished atoms $\left(A_{0}, B_{0}\right) \in \Sigma_{\mathrm{a}} \times \Sigma_{\mathrm{b}}$. In accordance with the previous point, it suffices to show that

$$
\min \left\{\left\|\mathrm{E}_{\Sigma_{\mathrm{a}}} \mathrm{E}_{\Sigma_{\mathrm{b}}} f\right\|_{2},\left\|\mathrm{E}_{\Sigma_{\mathrm{b}}} \mathrm{E}_{\Sigma_{\mathrm{a}}} f\right\|_{2}\right\} \leq c_{2}\left(\Sigma_{\mathrm{ab}}\right)\|f\|_{2}
$$

for some $0<c_{2}\left(\Sigma_{\mathrm{ab}}\right)<1$ and every mean-zero $f \in L_{2}(\Omega)$. We claim that this estimate follows if the same inequality holds for $\Sigma_{j}$-measurable functions which vanish on the corresponding distinguished atom. More precisely, it suffices to prove that one of the following conditions holds:

- $\left\|\mathrm{E}_{\Sigma_{\mathrm{a}}} \phi_{\mathrm{b}}\right\|_{2} \leq c_{2}\left(\Sigma_{\mathrm{ab}}\right)\left\|\phi_{\mathrm{b}}\right\|_{2}$ for $\phi_{\mathrm{b}} \Sigma_{\mathrm{b}}$-measurable with $\phi_{\mathrm{b}}\left(B_{0}\right)=0$;

- $\left\|\mathrm{E}_{\Sigma_{\mathrm{b}}} \phi_{\mathrm{a}}\right\|_{2} \leq c_{2}\left(\Sigma_{\mathrm{ab}}\right)\left\|\phi_{\mathrm{a}}\right\|_{2}$ for $\phi_{\mathrm{a}} \Sigma_{\mathrm{a}}$-measurable with $\phi_{\mathrm{a}}\left(A_{0}\right)=0$. 
Indeed, assume the first condition holds and let $\phi_{\mathrm{b}} \in L_{2}\left(\Omega, \Sigma_{\mathrm{b}}, \mu\right)$ be mean-zero. Then

$$
\begin{aligned}
\left\|\phi_{\mathrm{b}}\right\|_{2}^{2} & =\left\|\phi_{\mathrm{b}}-\phi_{\mathrm{b}}\left(B_{0}\right)\right\|_{2}^{2}-\left|\phi_{\mathrm{b}}\left(B_{0}\right)\right|^{2}, \\
\left\|\mathrm{E}_{\Sigma_{\mathrm{a}}} \phi_{\mathrm{b}}\right\|_{2}^{2} & =\left\|\mathrm{E}_{\Sigma_{\mathrm{a}}}\left(\phi_{\mathrm{b}}-\phi_{\mathrm{b}}\left(B_{0}\right)\right)\right\|_{2}^{2}-\left|\phi_{\mathrm{b}}\left(B_{0}\right)\right|^{2} .
\end{aligned}
$$

Subtracting and using the first condition, we get

$$
\left\|\phi_{\mathrm{b}}\right\|_{2}^{2}-\left\|\mathrm{E}_{\Sigma_{\mathrm{a}}} \phi_{\mathrm{b}}\right\|_{2}^{2} \geq\left(1-c_{2}\left(\Sigma_{\mathrm{ab}}\right)\right)\left\|\phi_{\mathrm{b}}-\phi_{\mathrm{b}}\left(B_{0}\right)\right\|_{2}^{2} \geq\left(1-c_{2}\left(\Sigma_{\mathrm{ab}}\right)\right)\left\|\phi_{\mathrm{b}}\right\|_{2}^{2}
$$

Here, $\phi_{\mathrm{b}}\left(B_{0}\right)$ denotes the constant value of $\phi_{\mathrm{b}}$ on $B_{0}$. Rearranging, we get $\left\|\mathrm{E}_{\Sigma_{\mathrm{a}}} \phi_{\mathrm{b}}\right\|_{2} \leq c_{2}\left(\Sigma_{\mathrm{ab}}\right)\left\|\phi_{\mathrm{b}}\right\|_{2}$. Therefore, given any mean-zero $f \in L_{2}(\Omega)$, we may define $\phi_{\mathrm{b}}=\mathrm{E}_{\Sigma_{\mathrm{b}}} f$ and deduce that $\left\|\mathrm{E}_{\Sigma_{\mathrm{a}}} \mathrm{E}_{\Sigma_{\mathrm{b}}} f\right\|_{2} \leq$ $c_{2}\left(\Sigma_{\mathrm{ab}}\right)\|f\|_{2}$, as desired. Alternatively, if we use the second condition above, the roles of $\Sigma_{\mathrm{a}}$ and $\Sigma_{\mathrm{b}}$ are switched and we obtain the other sufficient inequality which is implicit in the minimum above. Thus we have reduced the proof to justify one of the two conditions above. It is at this point where our definition of admissible pair comes into play. Namely, we know that

$$
\min \left\{\sup _{A \in \Pi_{\mathrm{a}} \backslash\left\{A_{0}\right\}} \sum_{B \in R_{A}}\left|R_{B}\right| \frac{\mu(A \cap B)^{2}}{\mu(A) \mu(B)}, \sup _{B \in \Pi_{\mathrm{b}} \backslash\left\{B_{0}\right\}} \sum_{A \in R_{B}}\left|R_{A}\right| \frac{\mu(A \cap B)^{2}}{\mu(A) \mu(B)}\right\}=c\left(\Sigma_{\mathrm{ab}}\right)
$$

for some $0<c\left(\Sigma_{\mathrm{ab}}\right)<1$. Let us assume (say) that the minimum above is attained by the first term and let $\phi_{\mathrm{a}}$ be a $\Sigma_{\mathrm{a}}$-measurable function in $L_{2}(\Omega)$ that vanishes on $A_{0}$. Then, if we write $\phi_{\mathrm{a}}=\sum_{A \neq A_{0}} \alpha_{A} \chi_{A}$, we have the estimate

$$
\begin{aligned}
\left\|\mathrm{E}_{\Sigma_{\mathrm{b}}} \phi_{\mathrm{a}}\right\|_{2}^{2} & =\sum_{A, A^{\prime} \neq A_{0}} \bar{\alpha}_{A} \alpha_{A^{\prime}} \sum_{B \in R_{A} \cap R_{A^{\prime}}} \frac{\mu(A \cap B)}{\mu(B)^{1 / 2}} \frac{\mu\left(A^{\prime} \cap B\right)}{\mu(B)^{1 / 2}} \\
& \leq \sum_{A, A^{\prime} \neq A_{0}} \frac{1}{2} \sum_{B \in R_{A} \cap R_{A^{\prime}}}\left(\left|\alpha_{A}\right|^{2} \frac{\mu(A \cap B)^{2}}{\mu(B)}+\left|\alpha_{A^{\prime}}\right|^{2} \frac{\mu\left(A^{\prime} \cap B\right)^{2}}{\mu(B)}\right) \\
& =\sum_{A \neq A_{0}}\left|\alpha_{A}\right|^{2} \mu(A) \sum_{\substack{A^{\prime} \neq A_{0} \\
R_{A} \cap R_{A^{\prime}} \neq \varnothing}} \sum_{B \in R_{A} \cap R_{A^{\prime}}} \frac{\mu(A \cap B)^{2}}{\mu(A) \mu(B)} \\
& =\sum_{A \neq A_{0}}\left|\alpha_{A}\right|^{2} \mu(A) \sum_{B \in R_{A}}\left|R_{B}\right| \frac{\mu(A \cap B)^{2}}{\mu(A) \mu(B)} \leq c\left(\Sigma_{\mathrm{ab}}\right) \sum_{A \neq A_{0}}\left|\alpha_{A}\right|^{2} \mu(A) .
\end{aligned}
$$

The right-hand side equals $c\left(\Sigma_{\mathrm{ab}}\right)\left\|\phi_{\mathrm{a}}\right\|_{2}^{2}$, so we obtain the second condition. The first one follows when the minimum in our definition of admissible covering is attained by the second term. This proves that the first assertion of Theorem A holds for finite measures and $p=2$. The case $p>2$ requires some preliminaries.

Step 4: A mass absorption principle. Let us consider a particular ordering of the atoms in $\Sigma_{\mathrm{a}}$ and $\Sigma_{\mathrm{b}}$. According to our assumption $\Sigma_{\mathrm{a}} \cap \Sigma_{\mathrm{b}}=\{\Omega, \varnothing\}$, we may order $\Pi_{\mathrm{a}}$ so that $\Pi_{\mathrm{a}}=\left\{A_{1}, A_{2}, \ldots\right\}$ and, for each $m \geq 0$, there exists $B \in \Pi_{\mathrm{b}}$ such that $\mu\left(A_{m+1} \cap B\right)$ and $\mu\left(\bigcup_{s \leq m} A_{s} \cap B\right)$ are both strictly positive. 
Similarly, we may order $\Pi_{b}$ satisfying the symmetric condition. Define the atomic $\sigma$-algebras

$$
\begin{aligned}
& \Sigma_{\mathrm{a}}(m)=\sigma\left\langle\bigcup_{s=0}^{m} A_{s},\left\{A_{s}\right\}_{s \geq m+1}\right\rangle, \\
& \Sigma_{\mathrm{b}}(m)=\sigma\left\langle\bigcup_{s=0}^{m} B_{s},\left\{B_{s}\right\}_{s \geq m+1}\right\rangle .
\end{aligned}
$$

In this step we will prove that

$$
\|f\|_{L_{\Sigma_{\mathrm{ab}}(\Omega)}^{p}} \simeq\left\|f-\mathrm{E}_{\Sigma_{\mathrm{a}}(m)} f\right\|_{L_{p}(\Omega)}+\left\|f-\mathrm{E}_{\Sigma_{\mathrm{b}}(m)} f\right\|_{L_{p}(\Omega)}
$$

for any $m \geq 1$ and $2<p<\infty$. The constants may depend on $m, p$ and the covering $\left(\Sigma_{\mathrm{a}}, \Sigma_{\mathrm{b}}\right)$. Indeed, since the result is trivial for $m=0$, we will proceed by induction and assume that the result holds for $m-1$. Moreover, the upper estimate is straightforward and by symmetry it suffices to show that

$$
\left\|f-\mathrm{E}_{\Sigma_{\mathrm{a}}(m)} f\right\|_{p} \lesssim\left\|f-\mathrm{E}_{\Sigma_{\mathrm{a}}(m-1)} f\right\|_{p}+\left\|f-\mathrm{E}_{\Sigma_{\mathrm{b}}} f\right\|_{p} .
$$

Taking $A_{0}(m)=\bigcup_{s \leq m} A_{s}$, let $f=f \chi_{A_{0}(m)}+f \chi_{\Omega \backslash A_{0}(m)}=f_{1}+f_{2}$. Since it is clear that $\mathrm{E}_{\Sigma_{\mathrm{a}}(m)} f_{2}=$ $\mathrm{E}_{\Sigma_{\mathrm{a}}(m-1)} f_{2}$, we may concentrate only on $f_{1}$. The left-hand side for $f_{1}$ can be written as

$$
\left\|f_{1}-\mathrm{E}_{\Sigma_{\mathrm{a}}(m)} f_{1}\right\|_{p}=\left\|\chi_{A_{0}(m)}\left(f-\mathrm{E}_{\Sigma_{\mathrm{a}}(m)} f\right)\right\|_{p}=\|f\|_{L_{p}^{\circ}\left(A_{0}(m)\right)} \sim \sup _{\substack{\|g\|_{L^{\prime}\left(A_{0}(m)\right)} \leq 1 \\ g \text { mean-zero }}}\left|\int_{A_{0}(m)} f g d \mu\right| .
$$

Approximating the right-hand side up to $\varepsilon>0$ by some mean-zero $g_{0}$ in the unit ball of $L_{p^{\prime}}\left(A_{0}(m)\right)$, let $B$ be an atom in $\Sigma_{\mathrm{b}}$ satisfying that $\mu\left(A_{0}(m-1) \cap B\right)$ and $\mu\left(A_{m} \cap B\right)$ are strictly positive. Recall that this can be done by the specific enumeration of atoms we picked. Then, define

$$
\begin{aligned}
& g_{1}=\chi_{A_{m}} g_{0}-\frac{\chi_{A_{m} \cap B}}{\mu\left(A_{m} \cap B\right)} \int_{A_{m}} g_{0} d \mu, \\
& g_{2}=\chi_{A_{0}(m-1)} g_{0}-\frac{\chi_{A_{0}(m-1) \cap B}}{\mu\left(A_{0}(m-1) \cap B\right)} \int_{A_{0}(m-1)} g_{0} d \mu, \\
& g_{3}=\frac{\chi_{A_{0}(m-1) \cap B}}{\mu\left(A_{0}(m-1) \cap B\right)} \int_{A_{0}(m-1)} g_{0} d \mu+\frac{\chi_{A_{m} \cap B}}{\mu\left(A_{m} \cap B\right)} \int_{A_{m}} g_{0} d \mu .
\end{aligned}
$$

Obviously, $g_{0}=g_{1}+g_{2}+g_{3}$ and each $g_{j}$ is mean-zero. Moreover, we have

$$
\begin{aligned}
\left\|g_{1}\right\|_{L_{p^{\prime}}\left(A_{0}(m)\right)} & \leq\left\|\chi_{A_{m}} g_{0}\right\|_{L_{p^{\prime}}\left(A_{0}(m)\right)}+\left\|\frac{\chi_{A_{m} \cap B}}{\mu\left(A_{m} \cap B\right)} \int_{A_{m}} g_{0} d \mu\right\|_{L_{p^{\prime}}\left(A_{0}(m)\right)} \\
& \leq\left(1+\frac{\mu\left(A_{m}\right)^{1 / p}}{\mu\left(A_{m} \cap B\right)^{1 / p}}\right)\left\|g_{0}\right\|_{L_{p^{\prime}}\left(A_{0}(m)\right)} \lesssim\left\|g_{0}\right\|_{L_{p^{\prime}}\left(A_{0}(m)\right)} \leq 1 .
\end{aligned}
$$


Similar computations apply to $g_{2}$ and $g_{3}$. In summary, we obtain the estimate below, where we write $f_{Q}$ to denote the average of $f$ over a given measurable set $Q$ :

$$
\begin{aligned}
\left\|f_{1}-\mathrm{E}_{\Sigma_{\mathrm{a}}(m)} f_{1}\right\|_{p} & \sim\left|\int_{A_{0}(m)} f g_{0} d \mu\right| \\
& \leq\left|\int_{A_{m}}\left(f-f_{A_{m}}\right) g_{1} d \mu\right|+\left|\int_{A_{0}(m-1)}\left(f-f_{A_{0}(m-1)}\right) g_{2} d \mu\right|+\left|\int_{B}\left(f-f_{B}\right) g_{3} d \mu\right| \\
& \lesssim\left\|\chi_{A_{m}}\left(f-f_{A_{m}}\right)\right\|_{p}+\left\|\chi_{A_{0}(m-1)}\left(f-f_{A_{0}(m-1)}\right)\right\|_{p}+\left\|\chi_{B}\left(f-f_{B}\right)\right\|_{p} \\
& \lesssim\left\|f-\mathrm{E}_{\Sigma_{\mathrm{a}}(m-1)} f\right\|_{p}+\left\|f-\mathrm{E}_{\Sigma_{\mathrm{b}}} f\right\|_{p} .
\end{aligned}
$$

This completes the proof of the norm equivalence (2-2).

Step 5: The case $\boldsymbol{p}>2$. We now complete the proof of Theorem A for probability measures. According to (2-2), it suffices to show that there exists $0<c_{p}\left(\Sigma_{\mathrm{ab}}\right)<1$ and $m=m(p) \geq 1$ such that, for any mean-zero function $f \in L_{p}(\Omega)$,

$$
\min \left\{\left\|\mathrm{E}_{\Sigma_{\mathrm{a}}(m)} \mathrm{E}_{\Sigma_{\mathrm{b}}(m)} f\right\|_{p},\left\|\mathrm{E}_{\Sigma_{\mathrm{b}}(m)} \mathrm{E}_{\Sigma_{\mathrm{a}}(m)} f\right\|_{p}\right\} \leq c_{p}\left(\Sigma_{\mathrm{ab}}\right)\|f\|_{p}
$$

Pick $m=m(p)$ as the smallest possible value of $m$ satisfying

$$
\min \left\{\mu\left(A_{0}(m)\right), \mu\left(B_{0}(m)\right)\right\}>\max \left\{\left(\frac{2 \cdot 4^{-p}}{1-2 \cdot 4^{-p}}\right)^{\frac{1}{p-1}},\left(1-4^{-p}\right)^{1 / p}\right\}
$$

and $\varepsilon=\varepsilon(p)>0$ small enough so that

$$
\left(1-2 \cdot 4^{-p}\right)^{1 / 2} \leq\left(1-4^{-p}\right)^{1 /(2 p)}\left(1-\varepsilon^{3}\right)^{1 / 2}-\varepsilon^{3 / 2}
$$

Since $L_{p}(\Omega) \subset L_{2}(\Omega)$, we know from Step 3 that $f$ always satisfies the above inequality for $p=2$. Assume that the minimum for $p=2$ is attained (say) at the first term, so that $\left\|\mathrm{E}_{\Sigma_{\mathrm{a}}(m)} \mathrm{E}_{\Sigma_{\mathrm{b}}(m)} f\right\|_{2} \leq c_{2}\left(\Sigma_{\mathrm{ab}}\right)\|f\|_{2}$. Recall that $\mathrm{E} f=\int_{\Omega} f d \mu$. When

$$
\mathrm{E}\left(\left|\mathrm{E}_{\Sigma_{\mathrm{b}}(m)} f\right|^{p / 2}\right)^{2}<\left(1-\varepsilon^{3}\right)\left\|\mathrm{E}_{\Sigma_{\mathrm{b}}(m)} f\right\|_{p}^{p}
$$

we proceed as follows:

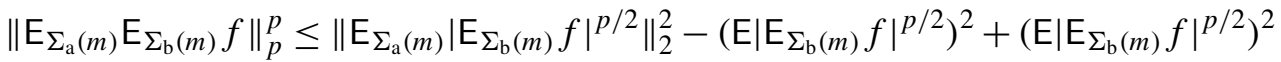

$$
\begin{aligned}
& =\left\|\mathrm{E}_{\Sigma_{\mathrm{a}}(m)}\left(\left|\mathrm{E}_{\Sigma_{\mathrm{b}}(m)} f\right|^{p / 2}-\left.\mathrm{E}_{\mid} \mathrm{E}_{\Sigma_{\mathrm{b}}(m)} f\right|^{p / 2}\right)\right\|_{2}^{2}+\left(\mathrm{E}\left|\mathrm{E}_{\Sigma_{\mathrm{b}}(m)} f\right|^{p / 2}\right)^{2} \\
& \leq c_{2}^{2}\left(\Sigma_{\mathrm{ab}}\right)\left\|\left|\mathrm{E}_{\Sigma_{\mathrm{b}}(m)} f\right|^{p / 2}-\left.\mathrm{E} \mathrm{E}_{\Sigma_{\mathrm{b}}(m)} f\right|^{p / 2}\right\|_{2}^{2}+\left(\left.\mathrm{E}_{\mid} \mathrm{E}_{\Sigma_{\mathrm{b}}(m)} f\right|^{p / 2}\right)^{2} \\
& \leq\left[c_{2}^{2}\left(\Sigma_{\mathrm{ab}}\right)+\left(1-c_{2}^{2}\left(\Sigma_{\mathrm{ab}}\right)\right)\left(1-\varepsilon^{3}\right)\right]\|f\|_{p}^{p}=c_{p}^{p}\left(\Sigma_{\mathrm{ab}}\right)\|f\|_{p}^{p} \text {. }
\end{aligned}
$$

If $\mathrm{E}\left(\left|\mathrm{E}_{\Sigma_{\mathrm{b}}(m)} f\right|^{p / 2}\right)^{2} \geq\left(1-\varepsilon^{3}\right)\left\|\mathrm{E}_{\Sigma_{\mathrm{b}}(m)} f\right\|_{p}^{p}$, then one can easily show that

$$
\left\|\left|\mathrm{E}_{\Sigma_{\mathrm{b}}(m)} f\right|^{p / 2}-\mathrm{E}\left|\mathrm{E}_{\Sigma_{\mathrm{b}}(m)} f\right|^{p / 2}\right\|_{2}^{2} \leq \varepsilon^{3}\left\|\mathrm{E}_{\Sigma_{\mathrm{b}}(m)} f\right\|_{p}^{p}
$$


Now, decomposing $\mathrm{E}_{\Sigma_{\mathrm{b}}(m)} f=\mathrm{E}_{\Sigma_{\mathrm{b}}(m)} f\left(B_{0}(m)\right) \chi_{B_{0}(m)}+\mathrm{E}_{\Sigma_{\mathrm{b}}(m)} f \chi_{\Omega \backslash B_{0}(m)}$, we get

$$
\begin{aligned}
\sqrt{\mu\left(B_{0}(m)\right)\left|\mathrm{E}_{\Sigma_{\mathrm{b}}(m)} f\left(B_{0}(m)\right)\right|^{p}} & =\left\|\left|\mathrm{E}_{\Sigma_{\mathrm{b}}(m)} f\right|^{p / 2} \chi_{B_{0}(m)}\right\|_{2} \\
& \geq\left\|\left.\mathrm{E}_{\mid} \mathrm{E}_{\Sigma_{\mathrm{b}}(m)} f\right|^{p / 2} \chi_{B_{0}(m)}\right\|_{2}-\|\left|\mathrm{E}_{\Sigma_{\mathrm{b}}(m)} f\right|^{p / 2}-\mathrm{E}_{\left|\mathrm{E}_{\Sigma_{\mathrm{b}}(m)} f\right|^{p / 2} \|_{2}} \\
& \geq \mu\left(B_{0}(m)\right)^{1 / 2} \mathrm{E}_{\left|\mathrm{E}_{\Sigma_{\mathrm{b}}(m)} f\right|^{p / 2}-\varepsilon^{3 / 2}\left\|\mathrm{E}_{\Sigma_{\mathrm{b}}(m)} f\right\|_{p}^{p / 2}} \\
& \geq\left[\left(1-4^{-p}\right)^{1 /(2 p)}\left(1-\varepsilon^{3}\right)^{1 / 2}-\varepsilon^{3 / 2}\right]\left\|\mathrm{E}_{\Sigma_{\mathrm{b}}(m)} f\right\|_{p}^{p / 2} \\
& \geq\left(1-2 \cdot 4^{-p}\right)^{1 / 2}\left\|\mathrm{E}_{\Sigma_{\mathrm{b}}(m)} f\right\|_{p}^{p / 2} .
\end{aligned}
$$

This also implies

$$
\left\|\mathrm{E}_{\Sigma_{\mathrm{b}}(m)} f \chi_{\Omega \backslash B_{0}(m)}\right\|_{p}^{p} \leq 2 \cdot 4^{-p}\left\|\mathrm{E}_{\Sigma_{\mathrm{b}}(m)} f\right\|_{p}^{p} .
$$

On the other hand, since $f$ is mean-zero we have

$$
\mathrm{E}_{\Sigma_{\mathrm{b}}(m)} f\left(B_{0}(m)\right) \mu\left(B_{0}(m)\right)+\mathrm{E}\left(\mathrm{E}_{\Sigma_{\mathrm{b}}(m)} f \chi_{\Omega \backslash B_{0}(m)}\right)=0 .
$$

Rearranging and raising to the power $p$ then gives

$$
\mu\left(B_{0}(m)\right)^{p}\left|\mathrm{E}_{\Sigma_{\mathrm{b}}(m)} f\left(B_{0}(m)\right)\right|^{p} \leq\left\|\mathrm{E}_{\Sigma_{\mathrm{b}}(m)} f \chi_{\Omega \backslash B_{0}(m)}\right\|_{p}^{p} \leq 2 \cdot 4^{-p}\left\|\mathrm{E}_{\Sigma_{\mathrm{b}}(m)} f\right\|_{p}^{p} .
$$

Finally, combining our two estimates so far for $\mu\left(B_{0}(m)\right)$, we obtain

$$
\mu\left(B_{0}(m)\right) \leq\left(\frac{2 \cdot 4^{-p}}{1-2 \cdot 4^{-p}}\right)^{\frac{1}{p-1}}
$$

which contradicts our choice of $m=m(p)$. This shows that $\mathrm{E}\left(\left|\mathrm{E}_{\Sigma_{\mathrm{b}}(m)} f\right|^{p / 2}\right)^{2}$ cannot be larger than $\left(1-\varepsilon^{3}\right)\left\|\mathrm{E}_{\Sigma_{\mathrm{b}}(m)} f\right\|_{p}^{p}$ and completes the proof in the case the minimum for $p=2$ is attained at the first term. When the minimum is attained at the second term, a symmetric argument applies.

Step 6: The nonfinite case. When $\mu(\Omega)=\infty$ the proof of Theorem A is a bit simpler. In the first place, note that $L_{p}^{\circ}(\Omega)=L_{p}(\Omega)$ in this case. In particular, the goal is to show that

$$
\begin{aligned}
& L_{q}(\Omega) \simeq\left[\operatorname{BMO}_{\Sigma_{\mathrm{ab}}}(\Omega), L_{1}(\Omega)\right]_{1 / q}, \\
& L_{p}(\Omega) \simeq L_{p}(\Omega, \Sigma, \mu) / \Sigma_{\mathrm{a}} \wedge L_{p}(\Omega, \Sigma, \mu) / \Sigma_{\mathrm{b}} .
\end{aligned}
$$

Since $L_{\infty}(\Omega) \subset \mathrm{BMO}_{\Sigma_{\mathrm{ab}}}(\Omega)$, our argument in Step 1 can be easily adapted and interpolation follows from the second isomorphism above. To prove it, we follow essentially the same argument as for finite measures. Indeed, arguing as in Step 2, we see that it suffices to show that

$$
\min \left\{\left\|\mathrm{E}_{\Sigma_{\mathrm{a}}} \mathrm{E}_{\Sigma_{\mathrm{b}}} f\right\|_{p},\left\|\mathrm{E}_{\Sigma_{\mathrm{b}}} \mathrm{E}_{\Sigma_{\mathrm{a}}} f\right\|_{p}\right\} \leq c_{p}\left(\Sigma_{\mathrm{ab}}\right)\|f\|_{p}
$$

for some constant $0<c_{p}\left(\Sigma_{\mathrm{ab}}\right)<1$ and every function $f \in L_{p}(\Omega)$. The only difference is that here it must hold for every $f$, not just mean-zero elements as in the finite case. The case $p=2$ is proved following Step 3. The fact that we do not assume $f$ to be mean-zero - or ultimately to vanish at $A_{0}$ or $B_{0}$ - is compensated by our definition of admissible coverings, which does not consider distinguished atoms 
for infinite measures. Finally, once we know the case $p=2$ holds — for arbitrary functions, not only mean-zero ones - we conclude that

$$
\left\|\mathrm{E}_{\Sigma_{\mathrm{a}}} \mathrm{E}_{\Sigma_{\mathrm{b}}} f\right\|_{p}^{p} \leq\left\|\mathrm{E}_{\Sigma_{\mathrm{a}}} \mathrm{E}_{\Sigma_{\mathrm{b}}}|f|^{p / 2}\right\|_{2}^{2} \leq c_{2}^{2}\left(\Sigma_{\mathrm{ab}}\right)\left\||f|^{p / 2}\right\|_{2}^{2} \leq c_{p}^{p}\left(\Sigma_{\mathrm{ab}}\right)\|f\|_{p}^{p}
$$

or a similar estimate for $\mathrm{E}_{\Sigma_{\mathrm{b}}} \mathrm{E}_{\Sigma_{\mathrm{a}}} f$. The proof of Theorem $\mathrm{A}$ is now complete.

\section{Calderón-Zygmund operators, I}

Let $(\Omega, \Sigma, \mu)$ be a measure space and consider a metric $d$ on $\Omega$. Assume that $\mu$ is $\sigma$-finite with respect to the metric topology. In this section we will be interested in Calderón-Zygmund operators on the metric measure space $(\Omega, \mu, d)$, as defined in the introduction. More precisely, we prove Theorem B1 below and, after that, we shall illustrate this result with a few constructions of admissible coverings.

Proof of Theorem B1. Our definition of CZO includes a symmetric Hörmander kernel condition. This implies that the class of Calderón-Zygmund operators is closed under taking adjoints. In particular, the $L_{p}$-boundedness for $1<p<2$ can be deduced by duality from the case $p>2$. On the other hand, according to Theorem A, the latter follows by interpolation if we can prove that any CZO extends to a bounded map $L_{\infty}(\Omega) \rightarrow \mathrm{BMO}_{\Sigma_{\mathrm{ab}}}(\Omega)$. Indeed, since $T$ is $L_{2}$-bounded, Theorem A yields that $T: L_{p}(\Omega) \rightarrow L_{p}^{\circ}(\Omega)$. This is enough when the measure $\mu$ is infinite, since in that case $L_{p}(\Omega)=L_{p}^{\circ}(\Omega)$. When $\mu$ is finite we use $L_{2}$-boundedness once again together with Hölder's inequality to deduce that

$$
\|T f\|_{p} \leq\|T f-\mathrm{E} T f\|_{p}+\mu(\Omega)^{1 / p}|\mathrm{E} T f| \lesssim\|f\|_{p}+\mu(\Omega)^{1 / p-1 / 2}\|f\|_{2} \lesssim\|f\|_{p} .
$$

This completes the proof of our claim. Let us then prove the $L_{\infty} \rightarrow$ BMO estimate. Consider an auxiliary BMO space which arises by averaging over the family of doubling balls in $(\Omega, \Sigma, \mu)$,

$$
\|f\|_{\mathrm{DBMO}}=\sup _{\substack{\mathrm{B} d-\mathrm{b} a l 1 \\ \text { doubling }}}\left(\frac{1}{\mu(\mathrm{B})} \int_{\mathrm{B}}\left|f(w)-\frac{1}{\mu(\mathrm{B})} \int_{\mathrm{B}} f d \mu\right|^{2} d \mu(w)\right)^{\frac{1}{2}} .
$$

Following the standard argument, it is easily checked that

$$
T: L_{\infty}(\Omega) \rightarrow \text { DBMO }
$$

Indeed, in the first place we may observe as usual that we have the equivalence

$$
\|f\|_{\mathrm{DBMO}} \sim \sup _{\substack{\mathrm{B} d \text {-ball } \\ \text { doubling }}} \inf _{\mathrm{B}} \in \mathbb{C}\left(\frac{1}{\mu(\mathrm{B})} \int_{\mathrm{B}}\left|f(w)-\mathrm{k}_{\mathrm{B}}\right|^{2} d \mu(w)\right)^{\frac{1}{2}} .
$$

Second, we decompose $f=f \chi_{\alpha \mathrm{B}}+f \chi_{\Omega \backslash \alpha \mathrm{B}}=\phi_{1 \mathrm{~B}}+\phi_{2 \mathrm{~B}}$ and pick the constant $\mathrm{k}_{\mathrm{B}}$ to be the average of $T \phi_{2 \mathrm{~B}}$ over B. Then, we may estimate the norm of $T f$ in DBMO by using the $L_{2}$-boundedness of $T$ for 
$T \phi_{1 \mathrm{~B}}$ and the Hörmander kernel condition for $T \phi_{2 \mathrm{~B}}$. More precisely, we get

$$
\begin{aligned}
\|T f\|_{\text {DBMO }} \leq \sup _{\substack{\mathrm{B} d \text {-ball } \\
\text { doubling }}}\left(\frac{1}{\mu(\mathrm{B})} \int_{\mathrm{B}} \mid\right. & \left.\left.T\left(f \chi_{\alpha \mathrm{B}}\right)(w)\right|^{2} d \mu(w)\right)^{\frac{1}{2}} \\
& +\left(\frac{1}{\mu(\mathrm{B})} \int_{\mathrm{B}}\left|T\left(f \chi_{\Omega \backslash \alpha \mathrm{B}}\right)(w)-\frac{1}{\mu(\mathrm{B})} \int_{\mathrm{B}} T\left(f \chi_{\Omega \backslash \alpha \mathrm{B}}\right) d \mu\right|^{2} d \mu(w)\right)^{\frac{1}{2}} .
\end{aligned}
$$

Since we just use $(\alpha, \beta)$-doubling balls, the first term is dominated by

$$
\left(\frac{\mu(\alpha \mathrm{B})}{\mu(\mathrm{B})}\right)^{\frac{1}{2}}\|T\|_{2 \rightarrow 2}\|f\|_{\infty} \lesssim\|f\|_{\infty} .
$$

On the other hand, using the kernel representation of $T$ we may write

$$
T\left(f \chi_{\Omega \backslash \alpha \mathrm{B}}\right)(w)-\frac{1}{\mu(\mathrm{B})} \int_{\mathrm{B}} T\left(f \chi_{\Omega \backslash \alpha \mathrm{B}}\right) d \mu=\frac{1}{\mu(\mathrm{B})} \int_{\mathrm{B}} \int_{\Omega \backslash \alpha \mathrm{B}}(k(w, \zeta)-k(\xi, \zeta)) f(\zeta) d \mu(\zeta) d \mu(\xi)
$$

for $w \in \mathrm{B}$. In particular, the last term above can be majorized by $\|f\|_{\infty}$ using the Hörmander condition for $k$. This proves the $L_{\infty}(\Omega) \rightarrow$ DBMO boundedness of our CZO. Therefore, it suffices to show that $\mathrm{DBMO} \subset \mathrm{BMO}_{\Sigma_{\mathrm{ab}}}(\Omega)$. This follows from the chain of inclusions

$$
\mathrm{DBMO} \subset \mathrm{bmo}_{\Sigma_{\mathrm{a}}} \wedge \mathrm{bmo}_{\Sigma_{\mathrm{b}}} \subset \mathrm{BMO}_{\Sigma_{\mathrm{a}}} \wedge \mathrm{BMO}_{\Sigma_{\mathrm{b}}}=\mathrm{BMO}_{\Sigma_{\mathrm{ab}}}(\Omega) .
$$

Let us recall in passing the terminology we are using, namely

$$
\mathrm{bmo}_{\Sigma_{j}}=J_{\Sigma_{j}}\left(\mathrm{bmo}_{j}\right) \quad \text { and } \quad \mathrm{BMO}_{\Sigma_{j}}=J_{\Sigma_{j}}\left(\mathrm{BMO}_{j}\right)
$$

for $j=\mathrm{a}, \mathrm{b}$. Here, $\mathrm{bmo}_{j}$ and $\mathrm{BMO}_{j}$ are the martingale bmo and $\mathrm{BMO}$ spaces constructed over the filtrations $\left(\Sigma_{j k}\right)_{k \geq 1}$ described in the statement of Theorem B1. If $\boldsymbol{\Pi}_{j}$ denotes the atoms in such a filtration, the norm in bmo $\Sigma_{j}$ is given by

$$
\|f\|_{\text {bmo }_{\Sigma_{j}}}=\sup _{k \geq 1}\left\|\mathrm{E}_{\Sigma_{j k}}\left|f-\mathrm{E}_{\Sigma_{j k}} f\right|^{2}\right\|_{\infty}^{1 / 2}=\sup _{A \in \Pi_{j}}\left(\frac{1}{\mu(A)} \int_{A}\left|f(w)-\frac{1}{\mu(A)} \int_{A} f d \mu\right|^{2} d \mu(w)\right)^{\frac{1}{2}} .
$$

Now, since we assume that all atoms in $\Pi=\Pi_{\mathrm{a}} \cup \Pi_{\mathrm{b}}$ are doubling, the seminorm above is majorized (up to absolute constants) by the seminorm in DBMO. As this holds for both $j=\mathrm{a}$, $\mathrm{b}$, we have proved the first inclusion. Now, for the second inclusion, we recall the seminorm in $\mathrm{BMO}_{\Sigma_{j}}$,

$$
\|f\|_{\mathrm{BMO}_{\Sigma_{j}}}=\sup _{k \geq 1}\left\|\mathrm{E}_{\Sigma_{j k}}\left|f-\mathrm{E}_{\Sigma_{j k-1}} f\right|^{2}\right\|_{\infty}^{1 / 2}
$$

where $\mathrm{E}_{\Sigma_{j 0}} f=\mathrm{E}_{\Sigma_{j 1}} f$ since we quotient out $\Sigma_{j 1}$-measurable functions. Note also that we are requiring the filtrations $\left(\Sigma_{j k}\right)_{k \geq 1}$ to be regular. In other words, there exist absolute constants $c_{j}>0$ such that $\mathrm{E}_{\Sigma_{j k}}|f| \leq c_{j} \mathrm{E}_{\Sigma_{j k-1}}|f|$ for $j=\mathrm{a}, \mathrm{b}$ and $k \geq 1$. This yields the inequality

$$
\|f\|_{\mathrm{BMO}_{\Sigma_{j}}} \leq c_{j}\|f\|_{\mathrm{bmo}_{\Sigma_{j}}} .
$$

Thus, $\mathrm{BMO}_{\Sigma_{\mathrm{ab}}}(\Omega) \simeq \mathrm{bmo}_{\Sigma_{\mathrm{a}}} \wedge \mathrm{bmo}_{\Sigma_{\mathrm{b}}}$ for regular filtrations, and we are done. 
Remark 3.1. Under the same assumptions, every CZO extends to a bounded map

$$
\mathrm{H}_{\Sigma_{\mathrm{ab}}}^{1}(\Omega) \rightarrow L_{1}(\Omega)
$$

Indeed, this follows at once by duality and Theorem B1. Alternatively, since we need to work with regular filtrations, we may use the atomic description of $\mathrm{H}_{\Sigma_{\mathrm{ab}}}^{1}(\Omega)$ given in Section 1, from which an easy argument arises; details are left to the reader.

In the following subsections we shall illustrate Theorem B1 with a few examples.

Doubling case. Admissible coverings fulfilling the assumptions in Theorem B1 can always be constructed on every doubling space, so that Calderón-Zygmund extrapolation for homogeneous spaces appears as a particular application of our approach. For clarity of the exposition, we shall just indicate how to construct such admissible coverings in $\mathbb{R}^{2}$ with the Lebesgue measure $m$ and the Euclidean metric, although a similar construction works in the general case. Let us pick $Q_{0}=\left[-\frac{1}{2}, \frac{1}{2}\right] \times\left[-\frac{1}{2}, \frac{1}{2}\right]$, the unit cube, and set $Q_{s}=3^{s} Q_{0}$ for $s \geq 1$. Consider the $\sigma$-algebras

$$
\Sigma_{\mathrm{a}}=\sigma\left\langle A_{s} \mid s \geq 1\right\rangle \quad \text { and } \quad \Sigma_{\mathrm{b}}=\sigma\left\langle B_{s} \mid s \geq 1\right\rangle,
$$

where $\left(A_{1}, B_{1}\right)=\left(Q_{0}, Q_{1}\right)$ and $\left(A_{s}, B_{s}\right)=\left(Q_{2 s-2} \backslash Q_{2 s-4}, Q_{2 s-1} \backslash Q_{2 s-3}\right)$ for $s \geq 2$. Then it follows from the proof of Lemma 1.3 that $\left(\Sigma_{\mathrm{a}}, \Sigma_{\mathrm{b}}\right)$ is an admissible covering of the Euclidean space $\left(\mathbb{R}^{2}, m\right)$.

Next, we define the filtrations $\left(\Sigma_{j k}\right)_{k \geq 1}$ with $\Sigma_{j 1}=\Sigma_{j}$ for $j=\mathrm{a}$, b. Except for $A_{1}$ and $B_{1}-$ which are ordinary cubes - the atoms $A_{s}$ and $B_{s}(s \geq 2)$ are punctured cubes in which we remove a concentric cube with side-length $\frac{1}{9}$ times the side-length of the larger one. To define $\Sigma_{j 2}$ for $j=\mathrm{a}$, b, we break each $A_{s}, B_{s}$ into a disjoint union of 80 equal cubes of side-length $\frac{1}{9}$ times the side-length of the original punctured cube - i.e., all except for the one in the center - unless $s=1$, in which case we also pick the center and get 81 subcubes (see Figure 1). The next generations are simpler. Indeed, since all our atoms in $\Sigma_{j 2}$ are already cubes, we perform dyadic partitions in each of them to provide the next generations of our filtration. This procedure completely defines two filtrations respectively based on $\Sigma_{\mathrm{a}}$ and $\Sigma_{\mathrm{b}}$. It remains to check that these filtrations are regular and the atoms are doubling. The regularity constant is dominated uniformly by 81 when $(k-1, k)=(1,2)$ and by 4 otherwise. On the other hand, our atoms for $k=1$ are punctured cubes which are comparable to the corresponding unpunctured ones, which in turn are doubling with constant 4 . This proves that all conditions in Theorem B1 are satisfied. In the general case, we just need to use Christ dyadic cubes [1990] and adapt our choice according to the finiteness or nonfiniteness of $\mu$ as we did in Lemma 1.3.

Polynomial growth. Assume that we have $(\Omega, \mu, d)$ of $k$-th degree polynomial growth with $\mu(\Omega)=\infty$. The associated RBMO norm can be defined as follows:

$$
\|f\|_{\mathrm{RBMO}}=\max \left\{\|f\|_{\mathrm{DBMO}}, \sup _{\substack{\mathrm{B} \subset \mathrm{B}^{\prime} \\ \mathrm{B}, \mathrm{B}^{\prime} d \text {-balls } \\ \text { doubling }}}\left|\frac{1}{\mu(\mathrm{B})} \int_{\mathrm{B}} f d \mu-\frac{1}{\mu\left(\mathrm{B}^{\prime}\right)} \int_{\mathrm{B}^{\prime}} f d \mu\right| / K_{\mathrm{B}, \mathrm{B}^{\prime}}\right\},
$$

with $1 \leq K_{\mathrm{B}, \mathrm{B}^{\prime}}=1+\sum_{2^{j} \mathrm{~B} \subset \mathrm{B}^{\prime}} \mu\left(2^{j} \mathrm{~B}\right) / r\left(2^{j} \mathrm{~B}\right)^{k}$. For such measures, we may easily construct an admissible covering of $(\Omega, \mu)$ composed of doubling atoms. Indeed, the construction above can easily be modified 


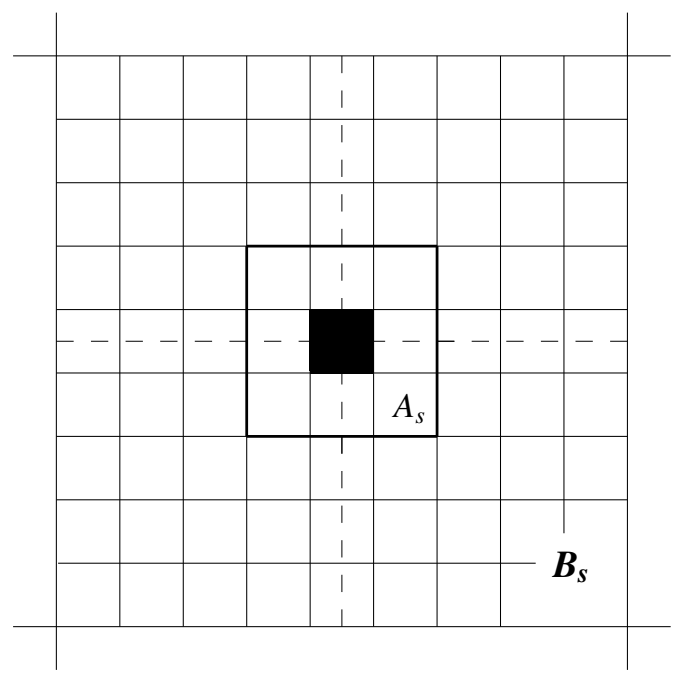

Figure 1. The admissible covering and the second generation of one of the filtrations.

using the existence of arbitrarily large doubling cubes centered at almost every point in the support of $\mu$; see [Tolsa 2001] for details. The main difficulty relies in the construction of filtrations $\left(\Sigma_{j k}\right)_{k \geq 1}$ satisfying the assumptions in Theorem B1. Note that, whenever that holds, we find

$$
\mathrm{RBMO} \subset \mathrm{DBMO} \subset \mathrm{BMO}_{\Sigma_{\mathrm{ab}}}(\Omega)
$$

In particular, we deduce that $\left[\mathrm{RBMO}, L_{1}(\Omega)\right]_{1 / q} \simeq L_{q}(\Omega)$ when this happens. As far as we know, such interpolation identities are new since Tolsa [2001] studied interpolation of operators. Unfortunately, the construction of such filtrations seems to be a difficult task in the general case. For instance, the corona-type construction described above finds some obstructions when the measure $\mu$ is supported in Cantor-like sets. Nevertheless, we may construct these filtrations in some other cases. Let us consider the following family of measures on $\mathbb{R}^{n}$ equipped with the Euclidean distance

$$
d \mu_{\beta}(x)=\frac{d x}{1+|x|^{\beta}} .
$$

These measures are nondoubling only for $\beta>n$. We will construct an admissible covering for $\beta \gtrsim n^{3 / 2}$ satisfying the hypotheses of Theorem B1 when $d$ is the Euclidean metric in $\mathbb{R}^{n}$. We will work with the equivalent measure

$$
d v_{\beta}(x)=\min \left\{1,|x|^{-\beta}\right\} d x
$$

for convenience. Note that this does not affect the conclusions in Theorem B1.

Pick $Q_{0}=[-\lambda, \lambda]^{n}$ with $\lambda>1$ to be fixed, and set $Q_{s}=2^{s} Q_{0}$. Consider the $\sigma$-algebras $\Sigma_{\mathrm{a}}=\sigma\left\langle A_{s}\right|$ $s \geq 1\rangle$ and $\Sigma_{\mathrm{b}}=\sigma\left\langle B_{s} \mid s \geq 1\right\rangle$, where $\left(A_{0}, B_{0}\right)=\left(Q_{0}, Q_{1}\right)$ and $\left(A_{s}, B_{s}\right)=\left(Q_{2 s} \backslash Q_{2 s-2}, Q_{2 s+1} \backslash Q_{2 s-1}\right)$ for any $s \geq 1$. We clearly have $\Sigma_{\mathrm{a}} \cap \Sigma_{\mathrm{b}}=\left\{\mathbb{R}^{n}, \varnothing\right\}$ and $\max \left\{\left|R_{A}\right|,\left|R_{B}\right|\right\} \leq 2$ for $(A, B) \in \Pi_{\mathrm{a}} \times \Pi_{\mathrm{b}}$, by 


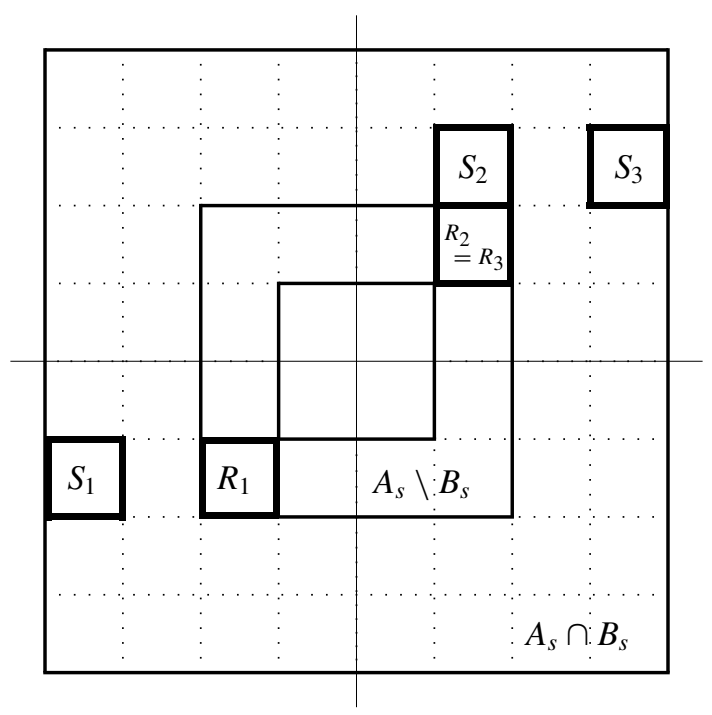

Figure 2. There is a cube $R_{j}$ for each cube $S_{j}$.

construction of $\left(\Sigma_{\mathrm{a}}, \Sigma_{\mathrm{b}}\right)$. Thus, it suffices to show that

$$
\sup _{\substack{(A, B) \in \Pi_{\mathrm{a}} \times \Pi_{\mathrm{b}} \\ A \neq A_{0}}} \frac{v_{\beta}(A \cap B)^{2}}{v_{\beta}(A) v_{\beta}(B)}<\frac{1}{2} .
$$

We will prove, in fact, the apparently stronger inequalities

$$
\sup _{s \geq 1} \frac{v_{\beta}\left(A_{s} \cap B_{s-1}\right)}{v_{\beta}\left(B_{s-1}\right)}<\frac{1}{2} \quad \text { and } \quad \sup _{s \geq 1} \frac{v_{\beta}\left(A_{s} \cap B_{s}\right)}{v_{\beta}\left(A_{s}\right)}<\frac{1}{2} .
$$

By symmetry of the argument, we just prove the second inequality above. Denote by $L$ the side length of the smallest cube $Q_{2 s}$ containing $A_{s}$. Then we have that $A \cap B$ can be decomposed into $\mathrm{C}_{n}=8^{n}-4^{n}$ cubes $S_{j}$, each of which satisfies that $S_{j}=R_{j}+a_{S_{j}}$ for some cube $R_{j}=R_{j}\left(S_{j}\right) \subset A_{s} \backslash B_{s}$ of side length equal to $L / 8$ and such that the angle between any point in $R_{j}$ and $a_{S_{j}}$ is smaller than $\pi / 3$. We can also impose that $\left|a_{S_{j}}\right| \geq L / 8$; see Figure 2. This implies that, for each $x$ in $R_{j}$, we have

$$
\left|x+a_{S_{j}}\right| \geq|x|+\left|a_{S_{j}}\right| \cos \varangle\left(x, a_{S_{j}}\right) \geq|x|+\frac{1}{2}\left|a_{S_{j}}\right| .
$$

Since $A_{s} \subset \mathbb{R}^{n} \backslash \mathrm{B}_{1}(0)$ for $s \geq 1$, we have

$$
\begin{aligned}
v_{\beta}\left(A_{s} \cap B_{s}\right) & =\int_{A_{s} \cap B_{s}}|x|^{-\beta} d x=\sum_{j=1}^{\mathrm{C}_{n}} \int_{S_{j}}|x|^{-\beta} d x \\
& =\sum_{j=1}^{\mathrm{C}_{n}} \int_{R_{j}}\left|x+a_{S_{j}}\right|^{-\beta} d x \leq \sum_{j=1}^{\mathrm{C}_{n}} \int_{R_{j}}\left(|x|+\frac{1}{2}\left|a_{S_{j}}\right|\right)^{-\beta} d x .
\end{aligned}
$$


Using that $|x| \leq \sqrt{n} L / 2$ for $x \in A_{s}$ and $\left|a_{S_{j}}\right| \geq L / 8$,

$$
\frac{1}{v_{\beta}\left(R_{j}\right)} \int_{R_{j}}\left(|x|+\frac{1}{2}\left|a_{S_{j}}\right|\right)^{-\beta} d x \leq \sup _{x \in R_{j}} \frac{\left(|x|+\frac{1}{2}\left|a_{S_{j}}\right|\right)^{-\beta}}{|x|^{-\beta}} \leq\left(\frac{\sqrt{n}}{\sqrt{n}+\frac{1}{8}}\right)^{\beta} .
$$

Therefore, we obtain

$$
\frac{v_{\beta}\left(A_{s} \cap B_{s}\right)}{v_{\beta}\left(A_{s}\right)} \leq \mathrm{C}_{n}\left(\frac{\sqrt{n}}{\sqrt{n}+\frac{1}{8}}\right)^{\beta} \leq 8^{n}\left(\frac{\sqrt{n}}{\sqrt{n}+\frac{1}{8}}\right)^{\beta}<\frac{1}{2} \quad \text { for } \beta \gtrsim n^{3 / 2} .
$$

A similar argument shows that

$$
\frac{v_{\beta}\left(A_{s} \cap B_{s-1}\right)}{v_{\beta}\left(B_{s-1}\right)}<\frac{1}{2}
$$

for $\beta \gtrsim n^{3 / 2}$ and $s \geq 2$, whereas the same estimate holds for $s=1$ as a consequence of the fact that $B_{0}$ contains $[-\lambda, \lambda]^{n}$ for $\lambda>1$ large enough. This completes the construction of an admissible covering. It remains to construct filtrations $\left(\Sigma_{j k}\right)_{k \geq 1}$ for $j=\mathrm{a}$, b, that are regular and composed of doubling atoms. Recall that we set $\Sigma_{j 1}=\Sigma_{j}$ and define $\Sigma_{j 2}$ by splitting each atom in $\Sigma_{j}$ into a disjoint union of cubes. Namely, for $j=$ a we keep $A_{0}$ and divide $A_{s}$ into the cubes $R_{j}, S_{j}$ in Figure 2. We proceed similarly for $j=\mathrm{b}$. Once we have defined $\Sigma_{j 2}$, we construct $\Sigma_{j k}$ by dyadic splitting of the cubes in $\Sigma_{j(k-1)}$. Note that the atoms in $\Sigma_{j 1} \backslash\left\{A_{0}, B_{0}\right\}$ split at most into $8^{n}$ cubes $K$ centered at $c_{K}$ which are away from the origin. Thus

$$
v_{\beta}(2 K)=\int_{2 K}|x|^{-\beta} d x \lesssim|2 K|\left|c_{K}\right|^{-\beta} \lesssim|K|\left|c_{K}\right|^{-\beta} \lesssim \int_{K}|x|^{-\beta} d x=v_{\beta}(K) .
$$

It easily follows from this that all the atoms in $\Sigma_{j k}$ are doubling up to absolute constants independent of $k \geq 1$ and that both filtrations are regular. This shows that Theorem B1 applies to $\left(\mathbb{R}^{n}, \mu_{\beta}\right)$ with the Euclidean metric.

Remark 3.2. A few comments are in order:

(i) In the light of the example above, one could wonder what happens with the positive powers $d \mu_{\gamma}(x)=$ $|x|^{\gamma} d x$ for $\gamma>0$, but it is straightforward to show that these measures are doubling, so that we can handle them following the construction of the previous section (see p. 731).

(ii) Our proof of Theorem B1 relies crucially on the embedding of the space DBMO in $\mathrm{BMO}_{\Sigma_{\mathrm{ab}}}(\Omega)$ under suitable conditions. When the metric measure space $(\Omega, \mu, d)$ is of polynomial growth, we know from [Tolsa 2001] that CZOs are $L_{\infty} \rightarrow$ RBMO bounded. Since RBMO $\subset$ DBMO, it is natural to wonder if we have

$$
\mathrm{RBMO} \subset \mathrm{BMO}_{\Sigma_{\mathrm{ab}}}(\Omega)
$$

under weaker assumptions than in Theorem B1. It turns out that this is the case when there exists filtrations composed of doubling atoms, no matter whether they are regular or not. Indeed, noticing that RBMO can be described as a subspace of DBMO with an additional condition, it is this crucial extra condition introduced by Tolsa that allows an embedding into $\mathrm{BMO}_{\Sigma_{\mathrm{ab}}}(\Omega)$ and not into bmo ${ }_{\Sigma_{\mathrm{ab}}}(\Omega)$ for nonregular filtrations. 
Concentration at the boundary. Let

$$
d \mu_{ \pm \alpha}(x)=e^{ \pm|x|^{\alpha}} d x
$$

on $\mathbb{R}^{n}$ equipped with the Euclidean metric. Carbonaro et al. [2009; 2010] proved that these measures satisfy their concentration condition when $\alpha>1$. In this subsection we shall prove that our hypotheses in Theorem B1 hold for any $\alpha>0$, hence extending their results for measures with less concentration at the boundary. Let us start with the probability measure $\mu_{-\alpha}$. Pick $K=K(n, \alpha)>0$, a large constant of the form $2^{k}$ for some $k \geq 1$ to be fixed below. Denote by $\boldsymbol{D}\left(\mathbb{R}^{n}\right)$ the standard filtration of dyadic cubes in $\mathbb{R}^{n}$. We consider the distinguished atom $A_{0}=[-K, K]^{n}$. The other atoms $A_{s} \in \Pi_{\mathrm{a}}$ for $s \geq 1$ are chosen to be the cubes in $\boldsymbol{D}\left(\mathbb{R}^{n} \backslash A_{0}\right)$ which are maximal under the following constraint on the side-length $\ell\left(A_{s}\right)$ in terms of the modulus of its center $c_{A_{s}}$ :

$$
\Pi_{\mathrm{a}}=\left\{A_{0}\right\} \cup\left\{A_{s} \text { maximal in }\left.\boldsymbol{D}\left(\mathbb{R}^{n} \backslash A_{0}\right)\left|\ell\left(A_{s}\right) \leq K\right| c_{A_{s}}\right|^{1-\alpha}, s \geq 1\right\} .
$$

Before defining $\Pi_{\mathrm{b}}$, we also need another dyadic filtration $\boldsymbol{D}^{\prime}\left(\mathbb{R}^{n}\right)$ satisfying some specific properties which we now detail. Given cubes $(A, B) \in \boldsymbol{D}\left(\mathbb{R}^{n}\right) \times \boldsymbol{D}^{\prime}\left(\mathbb{R}^{n}\right)$ of comparable size $-2^{-\mathrm{k}_{0}} \leq \ell(A) / \ell(B) \leq 2^{\mathrm{k}_{0}}$ for some absolute constant $\mathrm{k}_{0}$ - with nonempty intersection, there exists a parallelepiped $R \subset A \triangle B$ such that:

(1) $R$ is "substantially closer" than $A \cap B$ to the origin;

(2) there exists $a=a(R) \in \mathbb{R}^{n}$ such that $A \cap B \subset \bigcup_{j=1}^{\mathrm{N}} R+j a$;

(3) $|a| \geq \frac{1}{\mathrm{~N}} \max \{\ell(A), \ell(B)\}$ and $|x+j a| \geq|x|+\frac{1}{2}|a|$ for every $x \in R$.

Let $A_{1}$ be the cube in $\Pi_{\mathrm{a}} \backslash\left\{A_{0}\right\}$ whose center is the closest to the origin. Let $L=\ell\left(A_{1}\right)$ and pick $B_{0}=A_{0}+\frac{1}{3} L e_{d}$ with $e_{d}=(1,1, \ldots, 1)$. Then, the dyadic filtration $\boldsymbol{D}^{\prime}\left(\mathbb{R}^{n}\right)$ is defined as one of the shifted dyadic filtrations in [Conde 2013] with the initial cube being $B_{0}$. The fact that the properties above hold follows ultimately from the "good separation" between $\boldsymbol{D}\left(\mathbb{R}^{n}\right)$ and $\boldsymbol{D}^{\prime}\left(\mathbb{R}^{n}\right)$. Here we pick $K$ large enough so that the estimate $\mu_{-\alpha}\left(\frac{1}{2} A_{0}\right)>(1-\varepsilon) \mu_{-\alpha}\left(\mathbb{R}^{n}\right)$ holds. In particular, we get

$$
\frac{\mu_{-\alpha}\left(A_{0} \cap B_{0}\right)}{\mu_{-\alpha}\left(\mathbb{R}^{n}\right)}>1-\varepsilon
$$

for some small $\varepsilon>0$ to be fixed. The family $\Pi_{\mathrm{b}}$ is defined similarly by

$$
\Pi_{\mathrm{b}}=\left\{B_{0}\right\} \cup\left\{B_{s} \text { maximal in }\left.\boldsymbol{D}^{\prime}\left(\mathbb{R}^{n} \backslash B_{0}\right)\left|\ell\left(B_{s}\right) \leq K\right| c_{B_{s}}\right|^{1-\alpha}, s \geq 1\right\} .
$$

Set $\Sigma_{j}=\sigma\left(\Pi_{j}\right)$ for $j=\mathrm{a}, \mathrm{b}$ and observe that $\Sigma_{\mathrm{a}} \cap \Sigma_{\mathrm{b}}=\left\{\mathbb{R}^{n}, \varnothing\right\}$ by construction. Therefore, to prove that $\left(\Sigma_{\mathrm{a}}, \Sigma_{\mathrm{b}}\right)$ yields an admissible covering we only need to check that we have

$$
\sup _{A \in \Pi_{a} \backslash\left\{A_{0}\right\}} \sum_{B \in R_{A}}\left|R_{B}\right| \frac{\mu_{-\alpha}(A \cap B)^{2}}{\mu_{-\alpha}(A) \mu_{-\alpha}(B)}<1 .
$$

According to our definition of $A_{s}$, it is a simple exercise to check that we have $\ell\left(A_{s}\right) \geq \frac{1}{3} K\left|c_{A_{s}}\right|^{1-\alpha}$ for all $s \geq 1$ but for a finite number (independent of $K$ ) of cubes close to the origin. The same argument 


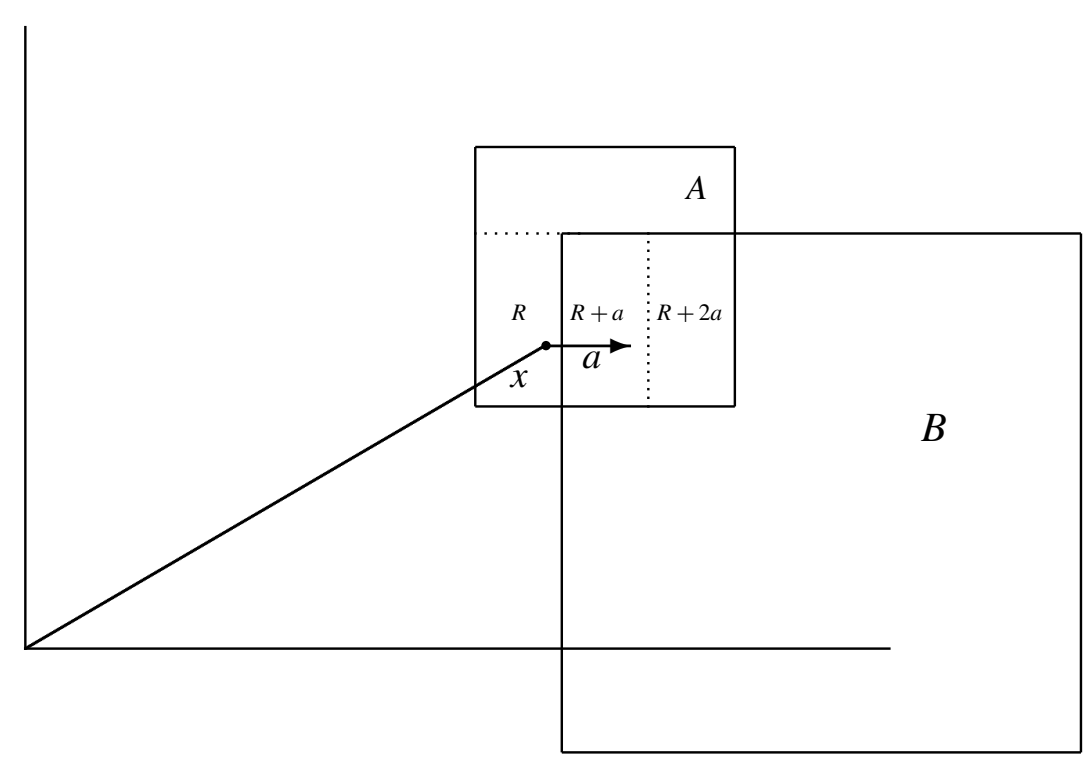

Figure 3. $A \cap B$ is covered by at most $\mathrm{N} a$-translates of $R ; R$ is "substantially closer" to the origin than $A \cap B ; a$ is parallel to a coordinate axis for cubes $A, B$ in a sector around that axis. In particular, $x$ and $a$ are "close" to being parallel, so that $|x+j a| \geq|x|+\frac{1}{2}|a|$.

holds for atoms in $\Pi_{\mathrm{b}}$. In particular, we have $\left|R_{A}\right|,\left|R_{B}\right| \leq \mathrm{C}_{n}$ for all $(A, B) \in \Pi_{\mathrm{a}} \times \Pi_{\mathrm{b}}$. Therefore, when $B=B_{0}$ we obtain

$$
\left|R_{B}\right| \frac{\mu_{-\alpha}(A \cap B)^{2}}{\mu_{-\alpha}(A) \mu_{-\alpha}(B)} \leq \mathrm{C}_{n} \frac{\mu_{-\alpha}(A \cap B)}{\mu_{-\alpha}(B)}<\mathrm{C}_{n} \frac{\varepsilon}{1-\varepsilon}<\frac{1}{2}
$$

for $\varepsilon<\frac{1}{3} \mathrm{C}_{n}^{-1}$. Otherwise, when $B \neq B_{0}$, we obtain

$$
\begin{aligned}
\frac{\mu_{-\alpha}(A \cap B)}{\mu_{-\alpha}(R)} & =\frac{1}{\mu_{-\alpha}(R)} \int_{A \cap B} e^{-|x|^{\alpha}} d x \\
& \leq \sum_{j=1}^{\mathrm{N}} \frac{1}{\mu_{-\alpha}(R)} \int_{R} e^{-|x+j a|^{\alpha}} d x \\
& \leq \frac{\mathrm{N}}{\mu_{-\alpha}(R)} \int_{R} e^{-(|x|+|a| / 2)^{\alpha}} d x \leq \mathrm{N} \sup _{x \in R} e^{-(|x|+|a| / 2)^{\alpha}+|x|^{\alpha}} .
\end{aligned}
$$

If $\alpha=1$, we get an estimate $\mathrm{N} e^{-|a| / 2} \geq \mathrm{N} e^{-\mathrm{C}_{n}^{\prime} K}$. For other values of $\alpha>0$, a straightforward application of the mean value theorem gives

$$
\left(|x|+\frac{1}{2}|a|\right)^{\alpha}-|x|^{\alpha} \geq \frac{\alpha}{18} K\left|c_{A}\right|^{1-\alpha}|x|^{\alpha-1} \geq \mathrm{C}_{n}^{\prime} K
$$

since $|x| \sim\left|c_{A}\right|$. Hence we get, for $A \neq A_{0}$,

$$
\sum_{B \in R_{A}}\left|R_{B}\right| \frac{\mu_{-\alpha}(A \cap B)^{2}}{\mu-\alpha(A) \mu-\alpha(B)}<\frac{1}{2}+\mathrm{C}_{n}^{2} \sup _{B \neq B_{0}} \frac{\mu_{-\alpha}(A \cap B)^{2}}{\mu_{-\alpha}(A) \mu_{-\alpha}(B)} \leq \frac{1}{2}+\mathrm{C}_{n}^{2} \mathrm{~N} e^{-\mathrm{C}_{n}^{\prime} K}<1,
$$


picking $K=K(n, \alpha)$ large enough. This shows that we have an admissible covering. Note that our choice of cubes for $\alpha>1$ is a family which becomes smaller and smaller when we get away from the origin. This is in the spirit of the Mauceri-Meda [2007] construction for the Gaussian measure. In contrast, when $\alpha<1$ we pick larger and larger cubes as we get away from the origin. This construction seems not to be useful in [Carbonaro et al. 2009; 2010], since we may not use the locally doubling property for arbitrarily large cubes. Let us complete the proof by showing that the other hypotheses in Theorem B1 hold. Our choice of filtrations $\left(\Sigma_{j k}\right)_{k \geq 1}$ for $j=\mathrm{a}$, b is by dyadic splitting of the cubes in $\Pi_{\mathrm{a}}$ and $\Pi_{\mathrm{b}}$, respectively. The regularity of such filtrations will follow from the fact that every atom in $\left(\Sigma_{j k}\right)_{k \geq 1}$ is (3, $\beta$ )-doubling for some absolute constant $\beta$, and this suffices to complete the proof. If $Q$ is any subcube of $A_{0} \cup B_{0}$, there are dimensional constants $k_{n}$ and $K_{n}$ such that

$$
k_{n}|Q| \leq \int_{Q} e^{-|x|^{\alpha}} d x \leq K_{n}|Q|,
$$

and hence $Q$ is trivially (3, $\beta$ )-doubling. Otherwise, we compute

$$
\begin{aligned}
\frac{\mu_{-\alpha}(3 Q)}{\mu_{-\alpha}(Q)} & \leq \frac{|3 Q|}{|Q|} \sup _{x \in 3 Q} e^{-|x|^{\alpha}} \sup _{x \in Q} e^{|x|^{\alpha}} \\
& \leq 3^{n} \exp \left(\left(\left|x_{Q}\right|+\frac{1}{2} \sqrt{n} \ell(Q)\right)^{\alpha}-\left(\left|x_{Q}\right|-\frac{3}{2} \sqrt{n} \ell(Q)\right)^{\alpha}\right) \leq \beta
\end{aligned}
$$

for some absolute constant $\beta>0$, using the mean value theorem one more time.

Remark 3.3. A few comments are in order:

(i) Given $\alpha>0$ and by minor modifications in the above arguments, we may also produce an admissible covering for $\left(\mathbb{R}^{n}, e^{|x|^{\alpha}} d x\right)$ which satisfies the hypotheses of Theorem B1 with respect to the Euclidean metric.

(ii) In this paper we $\wedge$-intersect two truncated martingale BMO spaces, but our results also hold for finite $\wedge$-intersections; details are simple and not very relevant. The Mauceri-Meda BMO space for the Gaussian measure [2007] can be described as such a finite intersection of BMO spaces using a construction similar to the one above for $\mu_{-2}$ but intersecting $n+1$ BMO spaces instead of 2. Namely, one uses as many filtrations as needed to cover all cubes in $\mathbb{R}^{n}$ with dyadic cubes of comparable size; see, for instance, [Conde 2013] for the optimal choice. This establishes an inclusion of their BMO space into our 2-intersection $\mathrm{BMO}_{\Sigma_{\mathrm{ab}}}$ associated to $\mu_{-2}$, which still interpolates and is strictly larger. The latter assertion can be proved following the argument which shows that classical BMO is strictly contained in dyadic BMO.

(iii) A geometric interpretation of our definition of admissible covering could be that we still impose certain concentration at the boundary, but much less than [Carbonaro et al. 2009; 2010]. In support of this, let us consider an admissible covering $\left(\Sigma_{\mathrm{a}}, \Sigma_{\mathrm{b}}\right)$. Let $\mathcal{A}$ be a finite family in $\Pi_{\mathrm{a}} \backslash\left\{A_{0}\right\}$ and let $R_{\mathcal{A}}$ be the union $\bigcup_{A \in \mathcal{A}} R_{A}$. If we consider the set $R_{\mathcal{A}}$ as a measurable set and interpret $R_{\mathcal{A}} \backslash \mathcal{A}$ as the region "close to the boundary", then we can prove that

$$
\mu\left(R_{\mathcal{A}}\right) \leq \frac{1}{1-c\left(\Sigma_{\mathrm{ab}}\right)} \mu\left(R_{\mathcal{A}} \backslash \mathcal{A}\right)
$$


or, equivalently, $\mu(\mathcal{A}) \leq c\left(\Sigma_{\mathrm{ab}}\right) \mu\left(R_{\mathcal{A}}\right)$. Indeed, we have

$$
\begin{aligned}
\mu(\mathcal{A}) & =\sum_{A \in \mathcal{A}} \sum_{B \in R_{\mathcal{A}}} \mu(A \cap B) \\
& \leq c\left(\Sigma_{\mathrm{ab}}\right)^{1 / 2} \sum_{A \in \mathcal{A}} \mu(A)^{1 / 2}\left(\sum_{B \in R_{\mathcal{A}}} \frac{\mu(B)}{\left|R_{B}\right|}\right)^{\frac{1}{2}} \\
& \leq c\left(\Sigma_{\mathrm{ab}}\right)^{1 / 2} \mu(\mathcal{A})^{1 / 2}\left(\sum_{A \in \mathcal{A}} \sum_{B \in R_{\mathcal{A}}} \frac{\mu(B)}{\left|R_{B}\right|}\right)^{\frac{1}{2}} \leq\left(c\left(\Sigma_{\mathrm{ab}}\right) \mu(\mathcal{A}) \mu\left(R_{\mathcal{A}}\right)\right)^{1 / 2} .
\end{aligned}
$$

(iv) In the case of the Gaussian measure on $\mathbb{R}^{n}$, Mauceri, Meda and Sjögren [Mauceri et al. 2012] proved that $R_{i}, S_{i}$, the Riesz transforms associated with the Ornstein-Uhlenbeck semigroups, are bounded from $L_{\infty}$ to Mauceri-Meda BMO spaces, but their adjoint operators $R_{i}^{*}, S_{i}^{*}$ are not when $n \geq 2$. As explained in (ii), our BMO spaces are strictly larger than Mauceri and Meda's if the $\sigma$-algebras $\Sigma_{a}, \Sigma_{b}$ are picked as in the beginning of this subsection. Therefore, the Riesz transforms $R_{i}, S_{i}$ studied in [Mauceri et al. 2012] are bounded from $L^{\infty}$ to our BMO spaces as well. Pierre Portal [2014] introduced a different type of Hardy spaces using truncated maximal functions and square functions. He proved that the Riesz transforms $R_{i}, S_{i}$ and their adjoint operators $R_{i}^{*}, S_{i}^{*}$ are all bounded from his $\mathrm{H}_{1}$ space to $L_{1}$ [Portal 2014, Theorem 6.1]. It is interesting to determine whether $R_{i}^{*}, S_{i}^{*}$ are $L^{\infty}$-BMO bounded for our BMO spaces with carefully picked $\Sigma_{a}, \Sigma_{b}$.

\section{Calderón-Zygmund operators, II}

In this section we will study the class of atomic Calderón-Zygmund operators (ACZO) defined in the introduction over a given measure space $(\Omega, \Sigma, \mu)$. More precisely, we shall prove Theorem B2 and illustrate it with a few constructions of dyadic operators satisfying its hypotheses.

Proof of Theorem B2. Following the same argument as in the proof of Theorem B1, we can use duality and our interpolation result in Theorem A to reduce the $L_{p}$-boundedness in the assertion to the $L_{\infty}(\Omega) \rightarrow \mathrm{BMO}_{\Sigma_{\mathrm{ab}}}(\Omega)$ boundedness of our ACZO. This is however standard. Indeed, since the filtration is regular we know that $\mathrm{BMO}_{\Sigma_{\mathrm{ab}}}(\Omega) \simeq \operatorname{bmo}_{\Sigma_{\mathrm{ab}}}(\Omega)$. Up to absolute constants, the norm in the latter space is given by

$$
\|T f\|_{\mathrm{bmo}_{\Sigma_{\mathrm{ab}}}(\Omega)}=\sup _{Q \in \Pi} \inf _{\mathrm{k}_{Q} \in \mathbb{C}}\left(\frac{1}{\mu(Q)} \int_{Q}\left|f(w)-\mathrm{k}_{Q}\right|^{2} d \mu(\omega)\right)^{\frac{1}{2}},
$$

where $\Pi=\Pi_{\mathrm{a}} \cup \boldsymbol{\Pi}_{\mathrm{b}}$ is the set of atoms in any of the two filtrations. Decompose

$$
f=f \chi_{\hat{Q}}+f \chi_{\Omega \backslash \hat{Q}}=f_{1}+f_{2}
$$

As usual, we pick $\mathrm{k}_{Q}=\left(T f_{2}\right)_{Q}$. Then, we control the term for $T f_{1}$ using the $L_{2}$-boundedness of $T$ and the regularity of the filtrations. The term $T f_{2}-\mathrm{k}_{Q}$ is dominated by means of the Hörmander kernel 
condition given in the definition of ACZO. Namely,

$$
\left(\frac{1}{\mu(Q)} \int_{Q}\left|T f_{1}(\omega)\right|^{2} d \mu(\omega)\right)^{\frac{1}{2}} \leq\|T\|_{2 \rightarrow 2} \sqrt{\frac{\mu(\hat{Q})}{\mu(Q)}}\|f\|_{\infty} \lesssim\|f\|_{\infty}
$$

by regularity of the filtrations. On the other hand

$$
\begin{aligned}
\left(\frac{1}{\mu(Q)} \int_{Q} \mid T f_{2}(\omega)-\right. & \left.\left.\left(T f_{2}\right)_{Q}\right|^{2} d \mu(\omega)\right)^{\frac{1}{2}} \\
\leq & \left.\leq \frac{1}{\mu(Q)^{2}} \int_{Q} \int_{Q}\left[\int_{\Omega \backslash \hat{Q}}\left|K\left(z_{1}, x\right)-K\left(z_{2}, x\right)\right| d \mu(x)\right]^{2} d \mu\left(z_{1}\right) d \mu\left(z_{2}\right)\right)^{\frac{1}{2}}\|f\|_{\infty}
\end{aligned}
$$

which is dominated by $\|f\|_{\infty}$ according to the Hörmander condition for ACZOs.

Remark 4.1. As mentioned in the introduction, standard prototypes of atomic Calderón-Zygmund operators include martingale transforms, perfect dyadic CZOs and Haar shift operators. These are usually defined on the Euclidean space $\mathbb{R}^{n}$ equipped with a dyadic filtration. Nevertheless, the exact same arguments apply on any dyadically doubling measure space or even for any measure space equipped with a two-sided regular filtration. López-Sánchez et al. [2014] have studied those nondoubling measure spaces for which Haar shift operators satisfy weak type- $(1,1)$ estimates. Theorem B2 provides a tool to produce nondoubling measure spaces over which Haar shifts, or more general atomic CZOs, are $L_{\infty} \rightarrow \mathrm{BMO}$ bounded. In the case of martingale transforms, Haar shift operators and perfect dyadic CZOs in $\left(\mathbb{R}^{n}, \mu\right)$, all of them satisfy the Hörmander-like condition

$$
\sup _{Q \in \boldsymbol{D}\left(\mathbb{R}^{n}\right)} \sup _{z_{1}, z_{2} \in Q} \int_{\mathbb{R}^{n} \backslash \hat{Q}}\left|k\left(z_{1}, x\right)-k\left(z_{2}, x\right)\right|+\left|k\left(x, z_{1}\right)-k\left(x, z_{2}\right)\right| d \mu(x)<\infty .
$$

This means that these operators are ACZOs satisfying Theorem B2 as long as we can find an admissible covering $\left(\Sigma_{\mathrm{a}}, \Sigma_{\mathrm{b}}\right)$ and regular filtrations over it such that all the atoms are cubes in $\boldsymbol{D}\left(\mathbb{R}^{n}\right)$ or suitable unions of those. If we review our examples in Section 3, this is not the case for our construction for $d \mu_{ \pm \alpha}(x)=e^{ \pm|x|^{\alpha}} d x$. It is however quite simple to adapt our construction for

$$
d \mu_{\beta}(x)=\frac{d x}{1+|x|^{\beta}}
$$

so that it satisfies the hypotheses of Theorem B2. In particular, the Haar shift operators defined on $\left(\mathbb{R}^{n}, \mu_{\beta}\right)$ are $L_{\infty}\left(\mathbb{R}^{n}\right) \rightarrow \mathrm{BMO}_{\Sigma_{\mathrm{ab}}}\left(\mathbb{R}^{n}\right)$ bounded. It remains open to decide whether an admissible covering exists on the exponential measure spaces $\left(\mathbb{R}^{n}, \mu_{ \pm \alpha}\right)$ using only atoms associated to one and not two dyadic systems.

\section{Matrix-valued forms of our results}

In this section, we extend our main results to the context of operator-valued functions. Noncommutative forms of Calderón-Zygmund theory have been recently studied in [Junge et al. $\geq 2015$; Mei and Parcet 2009; Parcet 2009; Mei 2007]. There are however no specific results in the context of nondoubling metric 
measure spaces. Unfortunately, it seems difficult to extend the approach of [Tolsa 2001] or [Mauceri and Meda 2007] to the operator-valued or even the noncommutative setting, since their interpolation results rest on good- $\lambda$ inequalities which do not have a noncommutative analogue so far. On the other hand, the semicommutative approach in [Parcet 2009] is valid for doubling spaces, but again presents serious obstacles to be extended to the nondoubling setting. The crucial aspect of our approach is that it ultimately rests on martingale inequalities that have been successfully transferred to the noncommutative setting. Namely, after Pixier and Xu's [1997] seminal contribution on Burkholder-Gundy inequalities for noncommutative martingales, we find analogues of Doob's maximal inequalities, Gundy, Davis and atomic decompositions, Burkholder conditional square functions, John-Nirenberg inequalities, $L_{p} / \mathrm{BMO}$ interpolation results; see [Hong and Mei 2012; Junge 2002; Junge and Mei 2010; Junge and Musat 2007; Junge and Perrin 2014; Junge and Xu 2003; Mei 2007; Musat 2003; Parcet and Randrianantoanina 2006; Perrin 2009] and the references therein.

Let us briefly introduce the framework for our results in this section; we refer to [Parcet 2009, Section 1] for a rather complete review of the necessary background adapted to our necessities. We also refer the reader to Pisier and Xu's survey [2003] for more on noncommutative $L_{p}$ theory. Let $(\Omega, \Sigma, \mu)$ be a $\sigma$-finite measure space and consider any pair $(\mathcal{M}, \tau)$ given by a von Neumann algebra $\mathcal{M}$ equipped with a normal, semifinite, faithful trace $\tau$. This is sometimes called a noncommutative measure space. We will write $(\mathcal{R}, \varphi)$ to denote the von Neumann algebra generated by essentially bounded functions $f: \Omega \rightarrow \mathcal{M}$ equipped with the trace

$$
\varphi(f)=\int_{\Omega} \tau(f(\omega)) d \mu(\omega) .
$$

$\mathcal{R}$ is the von Neumann algebra tensor product $\mathcal{R}=L_{\infty}(\Omega) \bar{\otimes} \mathcal{M}$ and we may consider the corresponding noncommutative spaces $L_{p}(\mathcal{R}, \varphi)$. This semicommutative model is the context where we intend to generalize our main results. Apart from its own interest as an operator-valued model, it constitutes a first step towards further results for more general von Neumann algebras. In particular, as [Junge et al. 2014] demonstrates, certain fully noncommutative questions can be reduced to the semicommutative setting. Readers not familiar with von Neumann algebra theory are encouraged to read this section restricting their attention to matrix-valued functions. In other words, replace $\mathcal{M}$ by the algebra $M_{m}$ of $m \times m$ matrices and $\tau$ by the standard trace tr. The difficulties are similar in this case to in the general setting, as long as we provide results with constants independent of $m$. We also refer to [Parcet 2009] for a comparison between this model and the vector-valued setting, which differs substantially in the endpoint estimates.

The BMO spaces. First we review the definitions and results in Section 1 for the semicommutative setting described above. Given a filtration $\left(\Sigma_{k}\right)_{k \geq 1}$ of $(\Omega, \Sigma, \mu)$, we consider the conditional expectations

$$
f \mapsto \mathrm{E}_{\Sigma_{k}} \otimes \operatorname{id}_{\mathcal{M}}(f) \in \mathcal{R} \quad \text { for } f \in \mathcal{R}
$$

still denoted by $\mathrm{E}_{\Sigma_{k}}$. The martingale bmo and BMO norms are

$$
\begin{aligned}
\|f\|_{\text {bmo }} & =\max \left\{\|f\|_{\mathrm{bmo}_{\mathrm{c}}},\left\|f^{*}\right\|_{\mathrm{bmo}_{\mathrm{c}}}\right\}, \\
\|f\|_{\mathrm{BMO}} & =\max \left\{\|f\|_{\mathrm{BMO}_{\mathrm{c}}},\left\|f^{*}\right\|_{\mathrm{BMO}_{\mathrm{c}}}\right\},
\end{aligned}
$$


where the column norms are defined as in the commutative case, taking into account that we use $|x|^{2}=x^{*} x$ for any operator $x$ on a Hilbert space. The interpolation result $\left[\mathrm{BMO}, L_{1}(\mathcal{R})\right]_{1 / p} \simeq L_{p}(\mathcal{R})$ was proved by Musat [2003] for any semifinite von Neumann algebra $\mathcal{R}$. This is the noncommutative analogue of the Janson-Jones interpolation theorem. If we set $\|f\|_{\mathrm{h}_{p}^{\mathrm{r}}}=\left\|f^{*}\right\|_{\mathrm{h}_{p}^{\mathrm{c}}}$, where the norm in $\mathrm{h}_{p}^{\mathrm{c}}$ is defined as in the commutative case, then the noncommutative Hardy spaces have the form

$$
\mathrm{h}_{p}= \begin{cases}\mathrm{h}_{p}^{\mathrm{r}}+\mathrm{h}_{p}^{\mathrm{c}} & \text { if } 1 \leq p \leq 2, \\ \mathrm{~h}_{p}^{\mathrm{r}} \cap \mathrm{h}_{p}^{\mathrm{c}} & \text { if } 2 \leq p \leq \infty .\end{cases}
$$

This combination of row and column square functions is known to be the right one for $L_{p}$ inequalities, as was discovered for the first time with the noncommutative Khintchine inequalities [Lust-Piquard 1986; Lust-Piquard and Pisier 1991]. The interpolation result [bmo, $\left.\mathrm{h}_{1}\right]_{1 / p} \simeq \mathrm{h}_{p}$ was proved in [Bekjan et al. 2010] for noncommutative martingales. As in the commutative case, the projections $J_{\Sigma_{1}}=\mathrm{id}-\mathrm{E}_{\Sigma_{1}}$ are bounded on bmo, BMO, $L_{p}$ and $\mathrm{h}_{p}$, so that we will be working with these complemented subspaces which enjoy the same interpolation and duality properties as the original spaces. Note that the identity

$$
\begin{aligned}
\|f\|_{J_{\Sigma_{1}}\left(\mathrm{bmo}_{\mathrm{c}}\right)} & =\sup _{k \geq 1}\left\|\left(\mathrm{E}_{\Sigma_{k}}\left|f-\mathrm{E}_{\Sigma_{k}} f\right|^{2}\right)^{1 / 2}\right\|_{\mathcal{M}} \\
& =\sup _{A \in \Pi}\left\|\left(\frac{1}{\mu(A)} \int_{A}\left|f(w)-\frac{1}{\mu(A)} \int_{A} f d \mu\right|^{2} d \mu(w)\right)^{\frac{1}{2}}\right\|_{\mathcal{M}}
\end{aligned}
$$

still holds and we have $J_{\Sigma_{1}}$ (bmo) $\simeq J_{\Sigma_{1}}$ (BMO) for regular filtrations. Consider an admissible covering $\left(\Sigma_{\mathrm{a}}, \Sigma_{\mathrm{b}}\right)$ of $(\Omega, \Sigma, \mu)$ and any pair of filtrations $\left(\Sigma_{j k}\right)_{k \geq 1}$ with $\Sigma_{j 1}=\Sigma_{j}$ for $j=\mathrm{a}$, b. Denote by $\mathrm{BMO}_{\mathrm{a}}$ and $\mathrm{BMO}_{\mathrm{b}}$ the $\mathrm{BMO}$ spaces associated to these filtrations in the semicommutative algebra $\mathcal{R}$ and set

$$
\mathrm{BMO}_{\Sigma_{j}}(\mathcal{R})=J_{\Sigma_{j}}\left(\mathrm{BMO}_{j}\right) \quad \text { and } \quad \mathrm{BMO}_{\Sigma_{\mathrm{ab}}}(\mathcal{R})=\mathrm{BMO}_{\Sigma_{\mathrm{a}}}(\mathcal{R}) \wedge \mathrm{BMO}_{\Sigma_{\mathrm{b}}}(\mathcal{R})
$$

The John-Nirenberg inequalities, atomic descriptions of $\mathrm{H}_{1}$ and duality results have also been transferred to the context of noncommutative martingales [Bekjan et al. 2010; Hong and Mei 2012; Junge and Musat 2007; Pisier and Xu 1997] and we will not review these results here, since they will not play a crucial role.

The interpolation theorem. Let us now state the analogue of Theorem A in the operator-valued setting. As usual, we will write $L_{p}^{\circ}(\mathcal{R})$ for the subspace of mean-zero elements with respect to $\mu$. In the terminology we use for admissible coverings,

$$
L_{p}^{\circ}(\mathcal{R}) \simeq J_{\Sigma_{\mathrm{a}} \cap \Sigma_{\mathrm{b}}}\left(L_{p}(\mathcal{R})\right) .
$$

Theorem 5.1. Let $\left(\Sigma_{\mathrm{a}}, \Sigma_{\mathrm{b}}\right)$ be an admissible covering in $(\Omega, \Sigma, \mu)$ and consider the semicommutative space $\mathcal{R}=L_{\infty}(\Omega) \bar{\otimes} \mathcal{M}$. Then, for each $2 \leq p<\infty$, there exists a constant $c_{p} \geq 1$ such that

$$
L_{p}^{\circ}(\mathcal{R}) \simeq_{c_{p}} J_{\Sigma_{\mathrm{a}}}\left(L_{p}(\mathcal{R})\right) \wedge J_{\Sigma_{\mathrm{b}}}\left(L_{p}(\mathcal{R})\right) .
$$

In particular, we have by complex interpolation that

$$
\left[\mathrm{BMO}_{\Sigma_{\mathrm{ab}}}(\mathcal{R}), L_{1}^{\circ}(\mathcal{R})\right]_{1 / q} \simeq_{c_{q}} L_{q}^{\circ}(\mathcal{R}) \quad(1<q<\infty),
$$

with $\mathrm{BMO}_{\Sigma_{\mathrm{ab}}}(\mathcal{R})$ constructed with any two filtrations over $\left(\Sigma_{\mathrm{a}}, \Sigma_{\mathrm{b}}\right)$. 
Sketch of the proof. Thanks to the close connection with martingales, the proof is entirely parallel to the one given in the classical case. Indeed, combining standard facts from noncommutative $L_{p}$ theory with the martingale results reviewed in the previous subsection, it is a simple exercise to adapt our proof of Theorem A to the present case. The only subtle point is the inequality

$$
\left\|\mathrm{E}_{\Sigma_{\mathrm{a}}} \mathrm{E}_{\Sigma_{\mathrm{b}}} f\right\|_{p}^{p} \leq\left\|\mathrm{E}_{\Sigma_{\mathrm{a}}}\left|\mathrm{E}_{\Sigma_{\mathrm{a}}} f\right|^{p / 2}\right\|_{2}^{2}
$$

which is used in the last two steps of our argument. Namely, in the classical case this is due to the conditional Jensen's inequality $\phi\left(\mathrm{E}_{\Sigma_{k}} f\right) \leq \mathrm{E}_{\Sigma_{k}} \phi(f)$ for convex functions $\phi$. In contrast, its noncommutative form does not hold for all $p \geq 2$, since we need the operator-convexity of the function $\phi(x)=|x|^{\beta}$ for $\beta=p / 2$ and $x$ not necessarily positive. This is the case for $\beta \geq 2$, or equivalently $p \geq 4$, but it fails for $2 \leq p<4$. Note however that the ultimate goal in Steps 5 and 6 is to show that $\left\|\mathrm{E}_{\Sigma_{\mathrm{a}}} \mathrm{E}_{\Sigma_{\mathrm{b}}} f\right\|_{p} \leq c_{p}\left(\Sigma_{\mathrm{ab}}\right)\|f\|_{p}$ for some $0<c_{p}\left(\Sigma_{\mathrm{ab}}\right)<1$. To prove it, we observe that

$$
\begin{aligned}
\mathrm{E}_{\Sigma_{\mathrm{a}}}\left(g_{1}^{*} g_{2}\right) & =\xi_{k}\left(g_{1}\right)^{*} \xi_{k}\left(g_{2}\right), \\
\mathrm{E}_{\Sigma_{\mathrm{a}}} \mathrm{E}_{\Sigma_{\mathrm{b}}}\left(f_{1}^{*} f_{2}\right) & =\omega_{k}\left(f_{1}\right)^{*} \omega_{k}\left(f_{2}\right)
\end{aligned}
$$

for certain right $\mathcal{R}_{k}$-module maps $\xi_{k}, \omega_{k}: L_{q}(\mathcal{R}) \rightarrow C_{q}\left(L_{q}(\mathcal{R})\right)$ with $\mathcal{R}_{k}=\mathrm{E}_{\Sigma_{k}}(\mathcal{R})$. This follows from standard factorization properties of completely positive unital maps in terms of Hilbert modules; see, for instance, [Junge 2002]. Let us consider the polar decompositions $f=u|f|$ and $g=v|g|$ of $g=\mathrm{E}_{\Sigma_{\mathrm{b}}} f$. Then we can factorize $\mathrm{E}_{\Sigma_{\mathrm{a}}} \mathrm{E}_{\Sigma_{\mathrm{b}}} f$ in two ways:

$$
\begin{aligned}
\mathrm{E}_{\Sigma_{\mathrm{a}}} g & =\mathrm{E}_{\Sigma_{\mathrm{a}}}\left(v|g|^{1 / 2}|g|^{1 / 2}\right)=\xi_{k}\left(|g|^{1 / 2} v^{*}\right)^{*} \xi_{k}\left(|g|^{1 / 2}\right), \\
\mathrm{E}_{\Sigma_{\mathrm{a}}} \mathrm{E}_{\Sigma_{\mathrm{b}}} f & =\mathrm{E}_{\Sigma_{\mathrm{a}}} \mathrm{E}_{\Sigma_{\mathrm{b}}}\left(u|f|^{1 / 2}|f|^{1 / 2}\right)=\omega_{k}\left(|f|^{1 / 2} u^{*}\right)^{*} \omega_{k}\left(|f|^{1 / 2}\right) .
\end{aligned}
$$

This yields the estimates

$$
\begin{aligned}
\left\|\mathrm{E}_{\Sigma_{\mathrm{a}}} \mathrm{E}_{\Sigma_{\mathrm{b}}} f\right\|_{p} & \leq\left\|\xi_{k}\left(|g|^{1 / 2} v^{*}\right)\right\|_{2 p}\left\|\xi_{k}\left(|g|^{1 / 2}\right)\right\|_{2 p} \\
& \leq\left\|\xi_{k}\left(|g|^{1 / 2} v^{*}\right)^{*} \xi_{k}\left(|g|^{1 / 2} v^{*}\right)\right\|_{p}^{1 / 2}\left\|\xi_{k}\left(|g|^{1 / 2}\right)^{*} \xi_{k}\left(|g|^{1 / 2}\right)\right\|_{p}^{1 / 2} \\
& =\left\|\mathrm{E}_{\Sigma_{\mathrm{a}}}\left(v|g| v^{*}\right)\right\|_{p}^{1 / 2}\left\|\mathrm{E}_{\Sigma_{\mathrm{a}}}(|g|)\right\|_{p}^{1 / 2} \leq\|f\|_{p}^{1 / 2}\left\|\mathrm{E}_{\Sigma_{\mathrm{a}}}\left|\mathrm{E}_{\Sigma_{\mathrm{b}}} f\right|^{p / 2}\right\|_{2}^{1 / p},
\end{aligned}
$$

and

$$
\begin{aligned}
\left\|\mathrm{E}_{\Sigma_{\mathrm{a}}} \mathrm{E}_{\Sigma_{\mathrm{b}}} f\right\|_{p} & \leq\left\|\omega_{k}\left(|f|^{1 / 2} u^{*}\right)\right\|_{2 p}\left\|\omega_{k}\left(|f|^{1 / 2}\right)\right\|_{2 p} \\
& \leq\left\|\omega_{k}\left(|f|^{1 / 2} u^{*}\right)^{*} \omega_{k}\left(|f|^{1 / 2} u^{*}\right)\right\|_{p}^{1 / 2}\left\|\omega_{k}\left(|f|^{1 / 2}\right)^{*} \omega_{k}\left(|f|^{1 / 2}\right)\right\|_{p}^{1 / 2} \\
& =\left\|\mathrm{E}_{\Sigma_{\mathrm{a}}} \mathrm{E}_{\Sigma_{\mathrm{b}}}\left(u|f| u^{*}\right)\right\|_{p}^{1 / 2}\left\|\mathrm{E}_{\Sigma_{\mathrm{a}}} \mathrm{E}_{\Sigma_{\mathrm{b}}}(|f|)\right\|_{p}^{1 / 2} \leq\|f\|_{p}^{1 / 2}\left\|\mathrm{E}_{\Sigma_{\mathrm{a}}} \mathrm{E}_{\Sigma_{\mathrm{b}}}|f|^{p / 2}\right\|_{2}^{1 / p} .
\end{aligned}
$$

The last inequality in both estimates follows from the Kadison-Schwarz inequality for operator-convex functions, since $\phi(x)=x^{\beta}$ is operator-convex on $\mathbb{R}_{+}$for $\beta \geq 1$. The first estimate is the right one to use in Step 5 and the second one in Step 6.

The Calderón-Zygmund operators. We now consider Calderón-Zygmund operators in semicommutative algebras associated to operator-valued kernels. Our construction is standard; we refer to [Duoandikoetxea 2001; Junge et al. 2014; Rubio de Francia et al. 1986] for further details. Let us write $L_{0}(\mathcal{M})$ for the *-algebra of $\tau$-measurable operators affiliated with $\mathcal{M}$ and consider kernels $k:(\Omega \times \Omega) \backslash \Delta \rightarrow \mathcal{L}\left(L_{0}(\mathcal{M})\right)$ 
defined away from the diagonal $\Delta$ of $\Omega \times \Omega$ and which take values in linear maps on $\tau$-measurable operators. If $d$ is a metric in $\Omega$, the standard Hörmander kernel condition takes the same form in this setting when we replace the absolute value by the norm in the algebra $\mathcal{B}(\mathcal{M})$ of bounded linear operators acting on $\mathcal{M}$ :

$$
\sup _{\substack{\mathrm{B} d-\text { ball } \\ z_{1}, z_{2} \in \mathrm{B}}} \int_{\Omega \backslash \alpha \mathrm{B}}\left\|k\left(z_{1}, x\right)-k\left(z_{2}, x\right)\right\|_{\mathcal{B}(\mathcal{M})}+\left\|k\left(x, z_{1}\right)-k\left(x, z_{2}\right)\right\|_{\mathcal{B}(\mathcal{M})} d \mu(x)<\infty .
$$

Define a CZO in $(\mathcal{R}, \varphi, d)$ as any linear map $T$ satisfying the following properties:

- $T$ is bounded on $L_{\infty}\left(\mathcal{M} ; L_{2}^{r}(\Omega)\right)$,

$$
\left\|\left(\int_{\Omega} T f(x) T f(x)^{*} d \mu(x)\right)^{\frac{1}{2}}\right\|_{\mathcal{M}} \lesssim\left\|\left(\int_{\Omega} f(x) f(x)^{*} d \mu(x)\right)^{\frac{1}{2}}\right\|_{\mathcal{M}} .
$$

- $T$ is bounded on $L_{\infty}\left(\mathcal{M} ; L_{2}^{c}(\Omega)\right)$,

$$
\left\|\left(\int_{\Omega} T f(x)^{*} T f(x) d \mu(x)\right)^{\frac{1}{2}}\right\|_{\mathcal{M}} \lesssim\left\|\left(\int_{\Omega} f(x)^{*} f(x) d \mu(x)\right)^{\frac{1}{2}}\right\|_{\mathcal{M}} .
$$

- The kernel representation

$$
T f(x)=\int_{\Omega} k(x, y)(f(y)) d \mu(y) \text { holds for } x \notin \operatorname{supp}_{\Omega} f
$$

and some kernel $k:(\Omega \times \Omega) \backslash \Delta \rightarrow \mathbb{C}$ satisfying the Hörmander condition.

The first two conditions replace the usual $L_{2}$-boundedness; see [Junge et al. 2014] for explanations.

Theorem 5.2. Let $\left(\Sigma_{\mathrm{a}}, \Sigma_{\mathrm{b}}\right)$ be an admissible covering of $(\Omega, \Sigma, \mu)$. Assume that $\Sigma$ admits regular filtrations $\left(\Sigma_{j k}\right)_{k \geq 1}$ by successive refinement of $\Sigma_{j 1}=\Sigma_{j}$ for $j=\mathrm{a}, \mathrm{b}$ and that each atom in $\Sigma_{j k}$ is a $\left(\mathrm{C}_{0}, \alpha, \beta\right)$-doubling set for certain absolute constants $\mathrm{C}_{0}, \alpha, \beta>0$. Let $\mathrm{BMO}_{\Sigma_{\mathrm{ab}}}(\mathcal{R})$ denote the $\wedge$-intersection of the $\mathrm{BMO}$ spaces defined over these filtrations. Then, every $\mathrm{CZO}$ extends to a bounded map:

(i) $\mathrm{H}_{\Sigma_{\mathrm{ab}}}^{1}(\mathcal{R}) \rightarrow L_{1}(\mathcal{R})$;

(ii) $L_{\infty}(\mathcal{R}) \rightarrow \mathrm{BMO}_{\Sigma_{\mathrm{ab}}}(\mathcal{R})$;

(iii) $L_{p}^{\circ}(\mathcal{R}) \rightarrow L_{p}^{\circ}(\mathcal{R})$ for $1<p<\infty$.

Moreover, if $T$ is $L_{2}(\mathcal{R})$-bounded then $T: L_{p}(\mathcal{R}) \rightarrow L_{p}(\mathcal{R})$ for all $1<p<\infty$.

Proof. According to Theorem 5.1 (interpolation) and the semicommutative form of Remark 3.1 (duality), it turns out that $L_{\infty}(\mathcal{R}) \rightarrow \mathrm{BMO}_{\Sigma_{\mathrm{ab}}}(\mathcal{R})$ boundedness automatically implies $\mathrm{H}_{\Sigma_{\mathrm{ab}}}^{1}(\mathcal{R}) \rightarrow L_{1}(\mathcal{R})$ boundedness, as well as $L_{p}^{\circ}(\mathcal{R}) \rightarrow L_{p}^{\circ}(\mathcal{R})$ boundedness. Moreover, if $T$ is also $L_{2}$-bounded we may reproduce the argument given in the proof of Theorem B1 to obtain $L_{p}$-boundedness for all $1<p<\infty$. Let us then focus on the $L_{\infty} \rightarrow$ BMO boundedness. Define 
with $\|f\|_{\text {DBMO }_{\mathrm{r}}}=\left\|f^{*}\right\|_{\mathrm{DBMO}_{\mathrm{c}}}$ and

$$
\|f\|_{\text {DBMO }_{\mathrm{c}}}=\sup _{\substack{\mathrm{B} \text { ball } \\ d \text {-doubling }}}\left\|\left(\frac{1}{\mu(\mathrm{B})} \int_{\mathrm{B}}\left|f(w)-\frac{1}{\mu(\mathrm{B})} \int_{\mathrm{B}} f d \mu\right|^{2} d \mu(w)\right)^{\frac{1}{2}}\right\|_{\mathcal{M}} .
$$

As usual, we write $|x|^{2}$ for $x^{*} x$. The assertion follows from

$$
L_{\infty}(\mathcal{R}) \stackrel{T}{\longrightarrow} \mathrm{DBMO} \stackrel{\mathrm{id}}{\longrightarrow} \mathrm{bmo}_{\Sigma_{\mathrm{ab}}}(\mathcal{R}) \simeq \mathrm{BMO}_{\Sigma_{\mathrm{ab}}}(\mathcal{R}) .
$$

The boundedness of the chain above can be justified as in the proof of Theorem B1. Indeed, the analogies in the argument lead us to apply the new conditions which appear in our definition of semicommutative CZO; see [Junge et al. 2014].

Remark 5.3. Theorem $B 2$ also admits a straightforward generalization to the semicommutative setting. Again, our use of martingale techniques makes the proof entirely analogous, so that we think it would be too repetitive to include it here.

\section{Acknowledgements}

Conde-Alonso and Parcet are partially supported by the ERC StG-256997-CZOSQP, the Spanish grant MTM2010-16518 and by ICMAT Severo Ochoa Grant SEV-2011-0087 (Spain). Mei is partially supported by the NSF DMS-1266042.

\section{References}

[Bekjan et al. 2010] T. N. Bekjan, Z. Chen, M. Perrin, and Z. Yin, "Atomic decomposition and interpolation for Hardy spaces of noncommutative martingales”, J. Funct. Anal. 258:7 (2010), 2483-2505. MR 2011d:46131 Zbl 1198.46047

[Bergh and Löfström 1976] J. Bergh and J. Löfström, Interpolation spaces: an introduction, Grundlehren der Math. Wissenschaften 223, Springer, Berlin, 1976. MR 58 \#2349 Zbl 0344.46071

[Carbonaro et al. 2009] A. Carbonaro, G. Mauceri, and S. Meda, " $H^{1}$ and BMO for certain locally doubling metric measure spaces”, Ann. Sc. Norm. Super. Pisa Cl. Sci. (5) 8:3 (2009), 543-582. MR 2010j:42025 Zbl 1180.42008

[Carbonaro et al. 2010] A. Carbonaro, G. Mauceri, and S. Meda, " $H^{1}$ and BMO for certain locally doubling metric measure spaces of finite measure”, Colloq. Math. 118:1 (2010), 13-41. MR 2011b:42034 Zbl 1193.42076

[Christ 1990] M. Christ, "A $T(b)$ theorem with remarks on analytic capacity and the Cauchy integral", Colloq. Math. 60/61:2 (1990), 601-628. MR 92k:42020 Zbl 0758.42009

[Conde 2013] J. M. Conde, "A note on dyadic coverings and nondoubling Calderón-Zygmund theory", J. Math. Anal. Appl. 397:2 (2013), 785-790. MR 2979613 Zbl 1254.42021

[Conde-Alonso and Parcet 2014] J. M. Conde-Alonso and J. Parcet, “Atomic blocks for noncommutative martingales", preprint, 2014. arXiv 1407.5451

[Davis 1970] B. Davis, “On the integrability of the martingale square function”, Israel J. Math. 8 (1970), 187-190. MR 42 \#3863 Zbl 0211.21902

[Duoandikoetxea 2001] J. Duoandikoetxea, Fourier analysis, Graduate Studies in Mathematics 29, American Mathematical Society, Providence, RI, 2001. MR 2001k:42001 Zbl 0969.42001

[Rubio de Francia et al. 1986] J. L. Rubio de Francia, F. J. Ruiz, and J. L. Torrea, "Calderón-Zygmund theory for operator-valued kernels", Adv. in Math. 62:1 (1986), 7-48. MR 88f:42035 Zbl 0627.42008 
[Garnett and Jones 1982] J. B. Garnett and P. W. Jones, "BMO from dyadic BMO”, Pacific J. Math. 99:2 (1982), 351-371. MR 85d:42021 Zbl 0516.46021

[Garsia 1973] A. M. Garsia, Martingale inequalities: seminar notes on recent progress, W. A. Benjamin, Reading, MA-LondonAmsterdam, 1973. MR 56 \#6844 Zbl 0284.60046

[Hong and Mei 2012] G. Hong and T. Mei, "John-Nirenberg inequality and atomic decomposition for noncommutative martingales”, J. Funct. Anal. 263:4 (2012), 1064-1097. MR 2927404 Zbl 06071594

[Hytönen and Kairema 2012] T. Hytönen and A. Kairema, "Systems of dyadic cubes in a doubling metric space", Colloq. Math. 126:1 (2012), 1-33. MR 2901199 Zbl 1244.42010

[Janson and Jones 1982] S. Janson and P. W. Jones, "Interpolation between $H^{p}$ spaces: the complex method", J. Funct. Anal. 48:1 (1982), 58-80. MR 84c:46021 Zbl 0507.46047

[John and Nirenberg 1961] F. John and L. Nirenberg, "On functions of bounded mean oscillation”, Comm. Pure Appl. Math. 14 (1961), 415-426. MR 24 \#A1348 Zbl 0102.04302

[Junge 2002] M. Junge, "Doob's inequality for non-commutative martingales”, J. Reine Angew. Math. 549 (2002), 149-190. MR 2003k:46097 Zbl 1004.46043

[Junge and Mei 2010] M. Junge and T. Mei, "Noncommutative Riesz transforms—a probabilistic approach", Amer. J. Math. 132:3 (2010), 611-680. MR 2012b:46136 Zbl 1200.46056

[Junge and Musat 2007] M. Junge and M. Musat, “A noncommutative version of the John-Nirenberg theorem", Trans. Amer. Math. Soc. 359:1 (2007), 115-142. MR 2007m:46103 Zbl 1173.46046

[Junge and Perrin 2014] M. Junge and M. Perrin, Theory of $\mathscr{H}_{p}$-spaces for continuous filtrations in von Neumann algebras, Astérisque 362, 2014. MR 3241706

[Junge and Xu 2003] M. Junge and Q. Xu, "Noncommutative Burkholder/Rosenthal inequalities", Ann. Probab. 31:2 (2003), 948-995. MR 2004f:46078 Zbl 1041.46050

[Junge et al. 2014] M. Junge, T. Mei, and J. Parcet, "Smooth Fourier multipliers on group von Neumann algebras", Geom. Funct. Anal. 24:6 (2014), 1913-1980. MR 3283931

[Junge et al. $\geq 2015$ ] M. Junge, T. Mei, and J. Parcet, "Algebraic Calderón-Zygmund theory”. In preparation.

[López-Sánchez et al. 2014] L. D. López-Sánchez, J. M. Martell, and J. Parcet, "Dyadic harmonic analysis beyond doubling measures", Adv. Math. 267 (2014), 44-93. MR 3269175 Zbl 1304.42041

[Lust-Piquard 1986] F. Lust-Piquard, "Inégalités de Khintchine dans $C_{p}(1<p<\infty)$ ", C. R. Acad. Sci. Paris Sér. I Math. 303:7 (1986), 289-292. MR 87j:47032 Zbl 0592.47038

[Lust-Piquard and Pisier 1991] F. Lust-Piquard and G. Pisier, "Noncommutative Khintchine and Paley inequalities", Ark. Mat. 29:2 (1991), 241-260. MR 94b:46011 Zbl 0755.47029

[Mateu et al. 2000] J. Mateu, P. Mattila, A. Nicolau, and J. Orobitg, "BMO for nondoubling measures”, Duke Math. J. 102:3 (2000), 533-565. MR 2001e:26019 Zbl 0964.42009

[Mauceri and Meda 2007] G. Mauceri and S. Meda, "BMO and $H^{1}$ for the Ornstein-Uhlenbeck operator", J. Funct. Anal. 252:1 (2007), 278-313. MR 2008m:42024 Zbl 1136.46027

[Mauceri et al. 2012] G. Mauceri, S. Meda, and P. Sjögren, "Endpoint estimates for first-order Riesz transforms associated to the Ornstein-Uhlenbeck operator", Rev. Mat. Iberoam. 28:1 (2012), 77-91. MR 2904131 Zbl 1238.42005

[Mei 2003] T. Mei, "BMO is the intersection of two translates of dyadic BMO", C. R. Math. Acad. Sci. Paris 336:12 (2003), 1003-1006. MR 2004e:42037 Zbl 1032.42026

[Mei 2007] T. Mei, “Operator valued Hardy spaces”, Mem. Amer. Math. Soc. 188:881 (2007), vi+64. MR 2010d:46085

[Mei and Parcet 2009] T. Mei and J. Parcet, "Pseudo-localization of singular integrals and noncommutative Littlewood-Paley inequalities”, Int. Math. Res. Not. 2009:8 (2009), 1433-1487. MR 2010k:46063 Zbl 1175.46057

[Musat 2003] M. Musat, "Interpolation between non-commutative BMO and non-commutative $L_{p}$-spaces", J. Funct. Anal. 202:1 (2003), 195-225. MR 2004g:46081 Zbl 1042.46038

[Nazarov et al. 2002] F. Nazarov, S. Treil, and A. Volberg, "Accretive system Tb-theorems on nonhomogeneous spaces", Duke Math. J. 113:2 (2002), 259-312. MR 2003g:42030 Zbl 1055.47027 
[Parcet 2009] J. Parcet, "Pseudo-localization of singular integrals and noncommutative Calderón-Zygmund theory", J. Funct. Anal. 256:2 (2009), 509-593. MR 2010g:42035 Zbl 1179.46051

[Parcet and Randrianantoanina 2006] J. Parcet and N. Randrianantoanina, "Gundy's decomposition for non-commutative martingales and applications", Proc. London Math. Soc. (3) 93:1 (2006), 227-252. MR 2007b:46115 Zbl 1119.46049

[Perrin 2009] M. Perrin, "A noncommutative Davis' decomposition for martingales", J. Lond. Math. Soc. (2) 80:3 (2009), 627-648. MR 2011e:46104 Zbl 1225.46048

[Pisier and Xu 1997] G. Pisier and Q. Xu, "Non-commutative martingale inequalities", Comm. Math. Phys. 189:3 (1997), 667-698. MR 98m:46079 Zbl 0898.46056

[Pisier and Xu 2003] G. Pisier and Q. Xu, "Non-commutative $L^{p}$-spaces", pp. 1459-1517 in Handbook of the geometry of Banach spaces, II, edited by W. B. Johnson and J. Lindenstrauss, North-Holland, Amsterdam, 2003. MR 2004i:46095 Zbl 1046.46048

[Portal 2014] P. Portal, "Maximal and quadratic Gaussian Hardy spaces”, Rev. Mat. Iberoam. 30:1 (2014), 79-108. MR 3186932 Zbl 1290.42048

[Tolsa 2001] X. Tolsa, "BMO, $H^{1}$, and Calderón-Zygmund operators for non doubling measures", Math. Ann. 319:1 (2001), 89-149. MR 2002c:42029 Zbl 0974.42014

Received 9 Jul 2014. Revised 18 Jan 2015. Accepted 6 Mar 2015.

Jose M. CONDE-ALOnSO: jose.conde@icmat.es

, Instituto de Ciencias Matemáticas, CSIC-UAM-UC3M-UCM Consejo Superior de Investigaciones Científicas, C/ Nicolás Cabrera 13-15, 28049 Madrid, Spain

TAO MEI: mei@wayne.edu

Department of Mathematics, Wayne State University, 656 W. Kirby St., Detroit, MI 48202, United States

and

Department of Mathematics, Baylor University, 1311 S 5th St., Waco, TX 77401, United States

JAVIER PARCET: javier.parcet@icmat.es

, Instituto de Ciencias Matemáticas, CSIC-UAM-UC3M-UCM Consejo Superior de Investigaciones Cientí\{\}ficas,

C/ Nicolás Cabrera 13-15, 28049 Madrid, Spain 


\title{
Analysis \& PDE
}

\author{
msp.org/apde
}

\section{EDITORS}

EDITOR-IN-CHIEF

\author{
Maciej Zworski \\ zworski@math.berkeley.edu \\ University of California \\ Berkeley, USA
}

BOARD OF EDITORS

Nicolas Burq Université Paris-Sud 11, France

nicolas.burq@math.u-psud.fr

Sun-Yung Alice Chang Princeton University, USA

chang@math.princeton.edu

Michael Christ University of California, Berkeley, USA

mchrist@math.berkeley.edu

Charles Fefferman Princeton University, USA

cf@math.princeton.edu

Ursula Hamenstaedt Universität Bonn, Germany

ursula@math.uni-bonn.de

Vaughan Jones U.C. Berkeley \& Vanderbilt University vaughan.f.jones@vanderbilt.edu

Herbert Koch Universität Bonn, Germany koch@math.uni-bonn.de

Izabella Laba University of British Columbia, Canada ilaba@math.ubc.ca

Gilles Lebeau Université de Nice Sophia Antipolis, France lebeau@unice.fr

László Lempert Purdue University, USA lempert@math.purdue.edu

Richard B. Melrose Massachussets Institute of Technology, USA rbm@math.mit.edu

Frank Merle Université de Cergy-Pontoise, France Frank.Merle@u-cergy.fr

William Minicozzi II Johns Hopkins University, USA minicozz@math.jhu.edu

Werner Müller Universität Bonn, Germany mueller@math.uni-bonn.de
Yuval Peres

Gilles Pisier

Tristan Rivière

Igor Rodnianski

Wilhelm Schlag

Sylvia Serfaty

Yum-Tong Siu

Terence Tao

Michael E. Taylor

Gunther Uhlmann

András Vasy

Dan Virgil Voiculescu

Steven Zelditch
University of California, Berkeley, USA

peres@stat.berkeley.edu

Texas A\&M University, and Paris 6

pisier@math.tamu.edu

ETH, Switzerland

riviere@math.ethz.ch

Princeton University, USA

irod@math.princeton.edu

University of Chicago, USA

schlag@math.uchicago.edu

New York University, USA

serfaty@cims.nyu.edu

Harvard University, USA siu@math.harvard.edu

University of California, Los Angeles, USA tao@math.ucla.edu

Univ. of North Carolina, Chapel Hill, USA met@math.unc.edu

University of Washington, USA gunther@math.washington.edu

Stanford University, USA andras@math.stanford.edu

University of California, Berkeley, USA

dvv@math.berkeley.edu

Northwestern University, USA

zelditch@math.northwestern.edu

PRODUCTION

production@msp.org

Silvio Levy, Scientific Editor

See inside back cover or msp.org/apde for submission instructions.

The subscription price for 2015 is US \$205/year for the electronic version, and \$390/year ( $\$ 55$, if shipping outside the US) for print and electronic. Subscriptions, requests for back issues from the last three years and changes of subscribers address should be sent to MSP.

Analysis \& PDE (ISSN 1948-206X electronic, 2157-5045 printed) at Mathematical Sciences Publishers, 798 Evans Hall \#3840, c/o University of California, Berkeley, CA 94720-3840, is published continuously online. Periodical rate postage paid at Berkeley, CA 94704, and additional mailing offices.

APDE peer review and production are managed by EditFLOw ${ }^{\circledR}$ from MSP.

\section{PUBLISHED BY}

mathematical sciences publishers

nonprofit scientific publishing

http://msp.org/

(C) 2015 Mathematical Sciences Publishers 


\section{ANALYSIS \& PDE}

\section{Volume $8 \quad$ No. $3 \quad 2015$}

Inverse scattering with partial data on asymptotically hyperbolic manifolds

RAPHAEL HORA and ANTÔNIO SÁ BARRETO

Low temperature asymptotics for quasistationary distributions in a bounded domain

TONY LELIÈVRE and FRANCIS NIER

Dynamics of complex-valued modified KdV solitons with applications to the stability of 629 breathers

Miguel A. Alejo and Claudio Muñoz

$L^{p}$ estimates for bilinear and multiparameter Hilbert transforms

WEI DAI and GUOZHEN LU

Large BMO spaces vs interpolation

Jose M. Conde-Alonso, Tao Mei and JaVier Parcet

Refined and microlocal Kakeya-Nikodym bounds for eigenfunctions in two dimensions 